bioRxiv preprint doi: https://doi.org/10.1101/2020.06.14.150946; this version posted November 9,2020 . The copyright holder for this

\title{
Global epistasis emerges from a generic model of a complex trait
}

\author{
Gautam Reddy ${ }^{*}$ \\ NSF-Simons Center for Mathematical \& Statistical Analysis of Biology, Harvard University, Cambridge, MA 02138 \\ Michael M. Desa们 \\ NSF-Simons Center for Mathematical and Statistical Analysis of Biology, Harvard University, Cambridge, MA 02138 \\ Department of Organismic and Evolutionary Biology, \\ Harvard University, Cambridge, MA 02138, USA \\ Quantitative Biology Initiative, Harvard University, Cambridge, MA 02138, USA and \\ Department of Physics, Harvard University, Cambridge, MA 02138, USA.
}

(Dated: November 9, 2020)

\begin{abstract}
Epistasis between mutations can make adaptation contingent on evolutionary history. Yet despite widespread "microscopic" epistasis between the mutations involved, microbial evolution experiments show consistent patterns of fitness increase between replicate lines. Recent work shows that this consistency is driven in part by global patterns of diminishing-returns and increasing-costs epistasis, which make mutations systematically less beneficial (or more deleterious) on fitter genetic backgrounds. However, the mechanistic basis of this "global" epistasis remains unknown. Here we show that diminishing-returns and increasing-costs epistasis emerge generically as a consequence of pervasive microscopic epistasis. Our model predicts a specific quantitative relationship between the magnitude of global epistasis and the stochastic effects of microscopic epistasis, which we confirm by re-analyzing existing data. We further show that the distribution of fitness effects takes on a universal form when epistasis is widespread, and introduce a novel fitness landscape model to show how phenotypic evolution can be repeatable despite sequence-level stochasticity.
\end{abstract}

Keywords: evolution, epistasis, complex trait, predictability

\section{INTRODUCTION}

Despite the idiosyncrasies of epistasis, a number of laboratory microbial evolution experiments show systematic patterns of convergent phenotypic evolution and declining adaptability. A striking example is provided by the E.coli long-term evolution experiment (LTEE) (Figure 17): 12 replicate populations that adapt in parallel show remarkably similar trajectories of fitness increase over time [1, 2], despite stochasticity in the identity of fixed mutations and the underlying dynamics of molecular evolution 3. 4. Similar consistent patterns of fitness evolution characterized by declining adaptability over time have also been observed in parallel yeast populations evolved from different genetic backgrounds and initial fitnesses [5] (Figure 10) and in other organisms [6-12. Declining adaptability is thought to arise from diminishing-returns epistasis [5, 13, 14, where a global coupling induced by epistatic interactions systematically reduces the effect size of individual beneficial mutations on fitter backgrounds. Diminishing-returns manifests as a striking linear dependence of the fitness effect of a mutation on background fitness (Figure 1 1 ). While diminishingreturns can be rationalized as the saturation of a trait

* gautam nallamala@fas.harvard.edu

† mdesai@oeb.harvard.edu close to a fitness peak, recent work shows a similar dependence on background fitness even for deleterious mutations, which become more costly on higher fitness backgrounds 15. This suggests that fitter backgrounds are also less robust to deleterious effects (Figure 11d), a phenomenon that has been termed increasing-costs epistasis. The mechanistic basis for the global coupling that results in these effects is unknown.

Put together, these observations suggest that the contributions to the fitness effect, $s_{i}$, of a mutation at a locus $i$ in a given genetic background can be written as

$$
s_{i}=s_{\text {additive }, i}+s_{\text {genotype }, i}-c_{i} y,
$$

where $s_{\text {additive }, i}$ is the additive effect of the mutation, $s_{\text {genotype } i} i$ is its genotype-dependent epistatic contribution independent of the background fitness $y$ (i.e., idiosyncratic epistasis), and $c_{i}$ quantifies the magnitude of global epistasis for locus $i$. Eq. (1) reflects the observation that the strength of global epistasis depends on the specific mutation and applies independently of whether its additive effect is deleterious (increasing-costs) or beneficial (diminishing-returns). Over the course of adaptation in a fixed environment, global epistatic feedback on mutational effects can lead to a long-term decrease in adaptability. If this feedback dominates, Eq. (1) suggests that the dependence of the fitness effect on evolutionary history is summarized entirely by the current fitness, and therefore results in predictable fitness evolution. 
Here, we show that diminishing-returns and increasing-costs epistasis are a simple consequence of widespread epistasis. This is consistent with recent work [16] that proposes a similar argument to explain these phenomena. However, while the core idea is similar, we present here an alternative framework based on the Fourier analysis of fitness landscapes, which leads to new insights and quantitative predictions. In particular, our framework leads to novel predictions for the relationship between the magnitude of global epistasis and the stochastic effects of microscopic epistasis, which we confirm by re-analyzing existing data. Extending this framework, we further quantify how the distribution of fitness effects shifts as the organism adapts and how the fitness effect of a mutation depends on the sequence of mutations that have fixed over the course of adaptation (i.e., historical contingency). While specific historical relationships depend on the genetic architecture, we introduce a novel fitness landscape model with an intuitive architecture for which the entire history is summarized by the current fitness. Using this fitness landscape model, we investigate the long-term dynamics of adaptation and elucidate the architectural features that lead to predictable fitness evolution.

\section{RESULTS}

\section{Diminishing-returns and increasing-costs epistasis}

We begin by examining the most general way to express the relationship between genotype and fitness (i.e., to describe the fitness landscape). A map between a quantitative trait (such as fitness), $y$, and the underlying genotype can be expressed as a sum of combinations of $\ell$ biallelic loci $x_{1}, x_{2}, \ldots, x_{\ell}$ that take on values $x_{i}= \pm 1$ :

$y=\bar{y}+\sum_{i} f_{i} x_{i}+\sum_{i>j} f_{i j} x_{i} x_{j}+\sum_{i>j>k} f_{i j k} x_{i} x_{j} x_{k}+\ldots$,

where $\bar{y}$ is a constant that sets the overall scale of fitness. The symmetric convention $x_{i}= \pm 1$ for the two allelic variants is less often used than $x_{i}=0,1$, but it is an equivalent formulation [17-21], which we employ here because it will prove more convenient for our purposes (see [22] for a discussion). The coefficients of terms linear in $x_{i}$ represent the additive contribution of each locus to the fitness (i.e. its fitness effect averaged across genotypes at all other loci), the higher-order terms quantify epistatic interactions of all orders, and $\bar{y}$ is the average fitness across all possible genotypes. Importantly, Eq. (2) makes apparent the idiosyncrasies induced by epistasis: a mutation at a locus with $\ell$ interacting partners has an effect composed of $2^{\ell-1}$ contributions.
To explicitly compute the fitness effect of a mutation at locus $i$ on a particular genetic background, we simply flip the sign of $x_{i}$, keeping all other $x_{j}$ constant, and write down the difference in fitness that results. This fitness effect will generally involve a sum over a large number of terms involving the f's in Eq. (2). While this may suggest that an analysis of fitness effects via Eq. (2) is intractable, the analysis in fact simplifies considerably if the locus has a significant number of independent interactions that contribute to the fitness (i.e., provided that the number of independent, nonzero epistatic terms associated to the locus is large). In this case, we show that the fitness effects of individual mutations decrease linearly with background fitness and the fluctuations around this linear trend are normally-distributed. In other words, Eq. (2) implies that widespread independent idiosyncratic epistatic interactions lead to the observed patterns of diminishing-returns and increasingcosts epistasis.

We present a derivation of this result in the SI. Here we explain the key intuition using a heuristic argument. The argument is based on a simple idea: for a welladapted organism $(y>\bar{y})$ with complex epistatic interactions, a mutation is more likely to disrupt rather than enhance fitness. To be quantitative, consider a highly simplified scenario where some number $N$ of the $f$ 's in Eq. (2) are \pm 1 at random and the others are 0 . In this case, the fitness of a given genotype is a sum of $N_{+}$and $N_{-}$interactions that contribute positively and negatively to the trait respectively, each with unit magnitude, so that $y=\bar{y}+N_{+}-N_{-}$. When positive and negative interactions balance, the organism is in a "neutrally-adapted" state $(y \approx \bar{y})$. By selecting for positive interactions, adaptation generates a bias so that $N_{+}>N_{-}$and $y>\bar{y}$. If locus $i$ involved in a fraction $v_{i}$ of all of $N=N_{+}+N_{-}$interactions is mutated, the effect of the mutation, on average, is to flip the sign of $N_{+} v_{i}$ positive interactions and $N_{-} v_{i}$ negative interactions. The new fitness is then $y_{i}=y-2 N_{+} v_{i}+2 N_{-} v_{i}=\bar{y}+\left(1-2 v_{i}\right)(y-\bar{y})$ and thus $s_{i}=y_{i}-y=-2 v_{i}(y-\bar{y})$. The negative linear relation between the background fitness, $y$, and the fitness effect of the mutation, $s_{i}$, is immediately apparent and emerges as a systematic trend simply due to a sampling bias towards positive interactions. Of course, while this relation is true on average, it is possible that locus $i$ affects more or less positive interactions due to sampling fluctuations. Provided only that $N$ is large and the interactions are independent, these fluctuations are approximately Gaussian with magnitude $\sqrt{N v_{i}\left(1-v_{i}\right)}$.

This basic argument holds beyond the simple model with unit interactions. In the more general case, if the mutation is directed from $x_{i}=-1 \rightarrow+1$, we show in the SI that its fitness effect, $s_{i}$, on a background of 

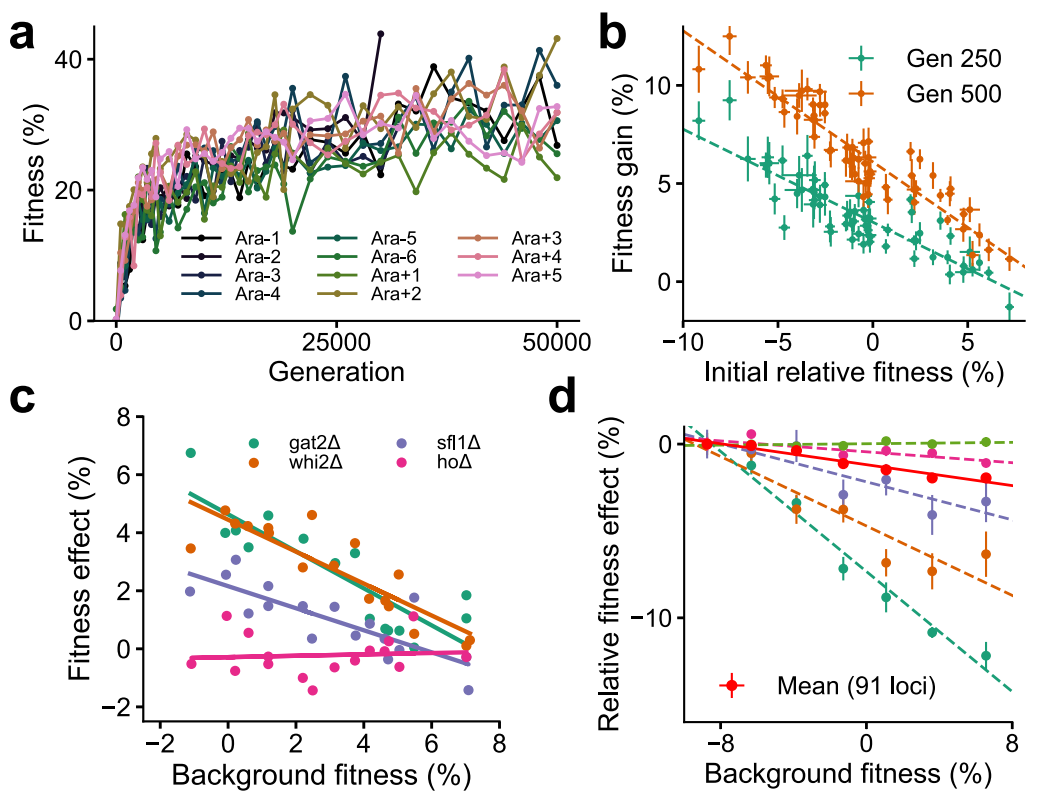

Figure 1. Declining adaptability and global epistasis in microbial evolution experiments. (a) Convergent phenotypic evolution in the E.coli long-term evolution experiment: the fitness relative to the common ancestor of 11 independently adapting populations over 50,000 generations is shown (data from [1]). The 12th population, Ara +6 , has limited data and is not shown. (b) Yeast strains with lower initial fitness adapt faster (data from [5]). The fitness gain after 250 (green) and 500 (orange) generations of 640 independently adapting populations with 64 different founders and 10 replicates of each founder. Mean and SE are computed over replicates. (c) Diminishing returns of specific beneficial mutations on fitter backgrounds for three knocked out genes (green, orange and purple) (data from [ $[$ ]). Control in pink. (d) Increasing costs of specific deleterious mutations on fitter backgrounds (data from [15]). The fitness effect relative to the least fit background for the mean over 91 mutations (in red) and five of the 91 mutations are shown. Linear fits for the five specific mutations and the mean using dashed and solid lines respectively are shown.

fitness $y$ can be written as

$$
s_{i}=\underbrace{2 f_{i}\left(1-\tilde{v}_{i}\right)}_{\text {additive }}-\underbrace{2 \tilde{v}_{i}(y-\bar{y})}_{\text {global epistasis }}+\underbrace{\tilde{\epsilon}_{i}}_{\text {genotype }}
$$

where

$\tilde{v}_{i} \equiv \frac{\sum_{j \neq i}\left(f_{i j}^{2}-f_{j} f_{i j}\right)+\sum_{j>k \neq i}\left(f_{i j k}^{2}-f_{j k} f_{i j k}\right)+\ldots}{\sum_{j \neq i}\left(f_{j}-f_{i j}\right)^{2}+\sum_{j>k \neq i}\left(f_{j k}-f_{i j k}\right)^{2}+\ldots}$,

and $\tilde{\epsilon}_{i}$ is a genotype and locus-dependent term which is distributed across genotypes with mean zero and variance expressed in terms of the $f$ 's from Eq. (2) (see SI for details). Note that $\tilde{v}_{i}$ depends on the direction of the mutation $x_{i}=-1 \rightarrow+1$; a similar equation for the case $x_{i}=+1 \rightarrow-1$ can be derived

Note that these results hold for any fitness landscape, and do not necessarily imply diminishing-returns or increasing-costs epistasis. That is, $\tilde{v}_{i}$ is not guaranteed to be positive, and $\tilde{\epsilon}_{i}$ is in general arbitrary and determined by the genotype-fitness map. However, consistent patterns emerge when locus $i$ has a large number of independent, nonzero epistatic terms and the additive effects $f_{1}, f_{2}, \ldots$ of its interacting partners are not much larger than the epistatic terms (defined further below), which we call the widespread-epistasis (WE) limit. In the WE limit, $\tilde{\epsilon}_{i}$ is normally-distributed across genotypes with variance proportional to $\tilde{v}_{i}\left(1-\tilde{v}_{i}\right)$. This follows from the same reasoning as in our heuristic argument with unit interactions above (see SI for details). In addition, $\tilde{v}_{i}$ is typically positive, giving rise to a negative linear trend (i.e. diminishing-returns and increasingcosts). We can see this by taking the third and higherorder terms in Eq. (4) to be zero, in which case $\tilde{v}_{i}$ is positive if $\sum_{j \neq i} f_{i j}^{2}>\sum_{j \neq i} f_{j} f_{i j}$. This will typically be true in the WE limit because we expect $\sum_{j \neq i} f_{i j}^{2}$ to scale with the number of interacting partners $\ell$, while each term in $\sum_{j \neq i} f_{j} f_{i j}$ can be positive or negative and thus the sum scales as $\sqrt{\ell}$ if the terms are independent. Thus when locus $i$ has a large number of interacting partners, $\tilde{v}_{i}$ is typically positive unless the magnitude of the additive terms $(a)$ is much larger than the magnitude of the epistatic terms $(e), a \gg e \sqrt{\ell}$. This argument is easily extended to the case when the third and higher-order terms are non-zero (see SI); the upshot is that the bias towards $\tilde{v}_{i}$ positive gets stronger with increasing epistasis. 
bioRxiv preprint doi: https://doi.org/10.1101/2020.06.14.150946; this version posted November 9, 2020. The copyright holder for this preprint (which was not certified by peer review) is the author/funder, who has granted bioRxiv a license to display the preprint in perpetuity. It is made available under aCC-BY-NC 4.0 International license.

The conditions for the WE limit are more likely to hold when the number of loci, $\ell$, that affect the trait is large. Therefore, we expect to generically observe patterns of diminishing-returns and increasing-costs epistasis for a complex trait involving many loci. Importantly, whether we observe a negative linear trend does not depend on the magnitude of a locus' epistatic interactions relative to its own additive effect, but rather relative to the additive effects of its interacting partners. If we are not in the WE limit, and instead the additive effects dominate (i.e., $a \gg e \sqrt{l}$ ), then Eq. (4) suggests that the slope of the linear trend can be either positive or negative. We will show further below that recent experimental data demonstrates that both scenarios can be relevant: some loci have $a \ll e \sqrt{l}$ while others have $a \gg e \sqrt{l}$, with the former creating a bias towards the observed negative linear trends that characterize diminishing-returns and increasing-costs epistasis.

We note that Eq. (3) immediately leads to testable quantitative predictions: in the WE limit, the distribution of the residuals, $\tilde{\epsilon}_{i}$, obtained from regressing $s_{i}$ and $y$ is entirely determined by the slope of the regression, $-2 \tilde{v}_{i}$. Specifically, we predict that these residuals (the deviations of individual genotype fitnesses from the overall diminishing-returns or increasing-costs trend) should be normally distributed with a variance proportional to $\tilde{v}_{i}\left(1-\tilde{v}_{i}\right)$. However, this condition only applies if diminishing-returns arises from the WE limit. It does not hold if epistasis is negligible, if locus $i$ interacts significantly with only a few other dominant loci, or if the epistatic terms are interrelated (e.g., when global epistasis arises from a nonlinearity applied to an unobserved additive trait $23-25]$ ). The latter case may still lead to a negative linear trend, but the statistics of the residuals will differ from Eq. (3) (see SI for a discussion).

It is convenient to subsequently work with the symmetric version of Eq. (3) where the fitness effects of both $x_{i}=-1 \rightarrow+1$ and $x_{i}=+1 \rightarrow-1$ are regressed against the background fitness. In this case, the additive term is averaged out, and in the WE limit we have

$$
s_{i}=-2 v_{i}(y-\bar{y})+2 \sqrt{v_{i}\left(1-v_{i}\right)} \eta_{i},
$$

where $\eta_{i}$ depends on the genetic background and the locus, and is normally-distributed with zero mean and variance $V$, and

$$
v_{i} \equiv \frac{V_{i}}{V}=\frac{f_{i}^{2}+\sum_{j \neq i} f_{i j}^{2}+\ldots}{\sum_{k} f_{k}^{2}+\sum_{k>l} f_{k l}^{2}+\ldots} .
$$

Here $V$ is the total genetic variance due to all loci (i.e., the variance in fitness across all possible genotypes) while $V_{i}$ is the contribution to the total variance by the $f$ 's involving locus $i$. We therefore refer to $v_{i}$ as the variance fraction of locus $i$. However, we emphasize that the $v_{i}$ 's do not sum to one unless there is no epistasis (with epistasis, $\sum_{i} v_{i}>1$, reflecting the fact that the variance contributed by different loci overlap). Eq. (5) and Eq. (3) coincide and $v_{i} \approx \tilde{v}_{i}$ in the WE limit if the additive effect of a locus is small (i.e., $\left.f_{i}^{2} \ll \sum_{j \neq i} f_{i j}^{2}+\sum_{j>k \neq i} f_{i j k}^{2}+\ldots\right)$.

Our results show that the variance fraction $v_{i}$ plays an important role. It determines the slope of the negative relationship between the fitness effect and background fitness. At the same time, it determines the magnitude of the idiosyncratic fluctuations away from this trend. We also note that this slope can be used to experimentally probe the contribution of a locus to the trait (i.e., its variance fraction) taking into account all orders of epistasis, which circumvents the estimation of the individual $f$ 's in Eq. (2). The theory additionally predicts that the negative slope of a double mutant at loci $i, j$ is $v_{i j}=v_{i}+v_{j}-2 e_{i j}$, where $e_{i j}$ quantifies the magnitude of epistatic interactions of all orders between $i$ and $j$ (SI).

Importantly, while the fitness effects of individual mutations (and hence the distribution of fitness effects) may change over the course of evolution due to epistasis, the distribution of variance fractions (DVF) across loci, $P(v)$, is an invariant measure of the range of effect-sizes available to the organism during adaptation. As we will see, this means that the DVF plays an important role in determining long-term adaptability.

\section{Numerical results and experimental tests}

To test our analytical results, we first demonstrate that the effects described above are reproduced in numerical simulations. To do so, we numerically generated a genotype-phenotype map of the form in Eq. (2), with $\ell=400$ loci and an exponential DVF, $P(v)=\bar{v}^{-1} e^{-v / \bar{v}}$, where $\bar{v}=0.02$ (Methods). This DVF is shown in Figure 2a. Note that $\bar{v} \ell \gg 1$ corresponds to an epistatic landscape; $\bar{v} \ell=8$ chosen here thus corresponds to a model within the WE limit (note that $\tilde{v}_{i} \approx v_{i}$ in this parameter range). Using this numerical landscape, we measured the fitness effect of mutations at 30 loci across 640 background genotypes with a range of fitnesses (Figure 20). Our results recapitulate the predicted linear dependence on background fitness (Figure $17, d)$, with a negative slope equal to twice the variance fraction predicted from Eq. (5). We further simulated the evolution of randomly generated genotypes similar to the experimental procedure used in Kryazhimskiy et al. 5] (Figure 2k), finding that our results reproduce the patterns of declining adaptability observed in experiments (Figure 1 b). Note that $\sim 10$ mutations are fixed during this simulated evolution; declining adaptability here is not due to a finite-sites effect. 
bioRxiv preprint doi: https://doi.org/10.1101/2020.06.14.150946; this version posted November 9, 2020. The copyright holder for this

As described previously, Eq. (5) implies a proportional relationship between the magnitude of global epistasis (quantified by the slope of the relationship between the fitness effect of a mutation and the background fitness) and the magnitude of microscopic epistasis (quantified by the residual variance around this linear trend); see also Figure 3a. We verify this relationship in simulations (Figure 2 $\mathrm{d}$ ). The negative slope of a double mutant at loci $i, j$ is predicted to be $v_{i j}=v_{i}+v_{j}-2 e_{i j}$. We further assume that $e_{i j}=O\left(\bar{v}^{2}\right)$ (specifically, $e_{i j}=v_{i} v_{j}$ for the genotypephenotype map used for numerics). Since $v_{i}$ and $v_{j}$ are typically small for a complex trait, we expect nearadditivity $v_{i j} \approx v_{i}+v_{j}$ and that any deviations are sub-additive, which is confirmed in simulations (Figure 2 e,f).

While testing the latter prediction on double mutants requires further experiments, we can immediately test the relationship between the slope and the distribution of residuals from existing experimental data. To do so, we re-analyzed the data from Johnson et al. [15], which measured the fitness effect of 91 insertion mutants on about 145 backgrounds. These background strains were obtained by crossing two yeast strains that differed by $\approx 40,000$ SNPs. Of these 40,000 loci, $\ell \approx 40$ have been identified as causal loci with currently available mapping resolution [26]. In Figure 3, we show the estimated $\tilde{v}_{i}$ (negative one-half of the slope of the best-fit line) and the variance fraction $v_{i}$ for each of the $91 \mathrm{mu}-$ tations. These mutations were selected after screening for nonzero effect, and thus the DVF is biased upwards. The mean variance fraction is $\bar{v} \approx 0.06$. The wide range of $v_{i}$ observed in the data implies that the epistatic influence of loci varies greatly across loci and we will show further below that this is crucial for maintaining a supply of beneficial mutations even when the organism is well-adapted to the environment.

Our theoretical results imply that we expect the linear relationship between background fitness and fitness effect to be negative if the additive effects of a locus' interacting partners are not much larger than the epistatic terms. Specifically, we define the additivity of interacting loci (AoIL) for locus $i$ as

$$
\begin{aligned}
\operatorname{AoIL}(i) & \equiv \frac{K}{1+K}, \quad \text { where } \\
K & =\frac{\left|\sum_{j \neq i} f_{j} f_{i j}+\sum_{j>k \neq i} f_{j k} f_{i j k}+\ldots\right|}{\sum_{j \neq i} f_{i j}^{2}+\sum_{j>k \neq i} f_{i j k}^{2}+\ldots}
\end{aligned}
$$

which we show can be estimated from data (Methods and SI). If the AoIL is less than half, Eq. (4) implies that the linear trend is guaranteed to be negative. If instead the AoIL is greater than 0.5, the trend can be either positive or negative. The data shows a range of AoIL between 0 and 1 across loci. As predicted by our theory, we find that the loci with AoIL $<0.5$ always show negative trends and the ones with AoIL $>0.5$ show both negative and positive trends (Figure 3r). Importantly, the sign of the trend is determined by the AoIL and not by the additivity of the mutated locus, which we define as

$$
\operatorname{Additivity}(i) \equiv \frac{f_{i}^{2}}{f_{i}^{2}+\sum_{j \neq i} f_{i j}^{2}+\sum_{j>k \neq i} f_{i j k}^{2}+\ldots} .
$$

The additivity across loci also has a wide range. However, small additivity does not necessarily imply a negative trend (Figure 3d).

We next used the data from Johnson et al. 15] to analyze the relationship between the slope of the linear trend and the residual variance around this trend. We find that the experimental data confirms our theoretical prediction that the residual variance is proportional to $\tilde{v}_{i}\left(1-\tilde{v}_{i}\right)$ if the AoIL is small (Figure 3e, $R^{2}=0.5$ for loci with AoIL $<0.5$ and $R^{2}=0.42$ for all loci). The Gaussian-distributed term in Eq. (3) also predicts the shape of the distribution of the residuals given the variance fractions, which aligns well with the empirical distribution of the residuals (Figure 3 ).

Together, these theoretical results and our reanalysis of experimental data show that linear patterns of global diminishing-returns and increasing-costs epistasis are a simple consequence of widespread epistatic interactions. The distribution of variance fractions observed in data (Figure 3b) further implies that the epistatic influence of different loci on fitness can vary across a wide range. In what follows, we show that these two observations can be put together to make general predictions about the distribution of fitness effects, and consequently the long-term dynamics of adaptation. The key ingredient that enables this analysis (including Eq. (5)) is that in the WE limit, fitness and fitness effects are jointly normal (with respect to a uniform distribution over all possible genotypes), which allows us to quantify complex dependencies between these variables in terms of pairwise covariances.

\section{The distribution of fitness effects}

Long-term adaptation is determined by the distribution of fitness effects (DFE) of possible mutations and the stochastic dynamical processes that lead to fixation. While Eq. (5) represents the distribution of the fitness effects of a specific mutation at locus $i$ over all genotypes in the population that have fitness $y$, we are instead interested in the DFE, where fitness effects are measured for all the mutations arising in the background of a particular genotype that has fitness $y$. For now we ignore the influence of evolutionary history on the DFE; we expand on that complication in the following Section. 
bioRxiv preprint doi: https://doi.org/10.1101/2020.06.14.150946; this version posted November 9, 2020. The copyright holder for this
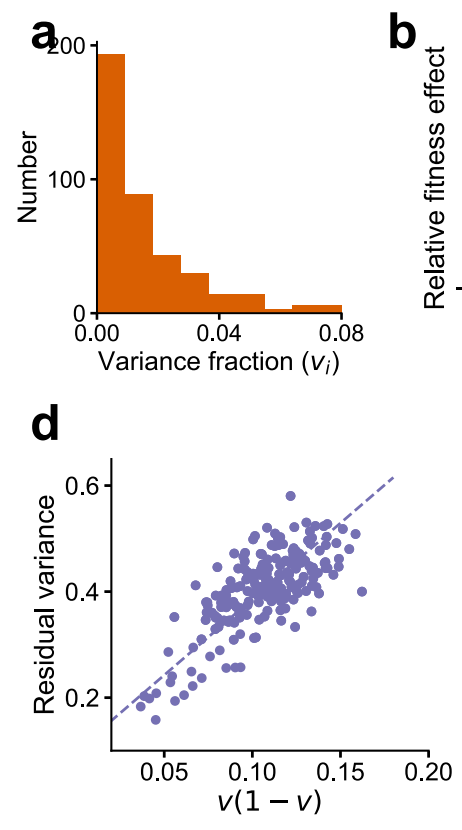
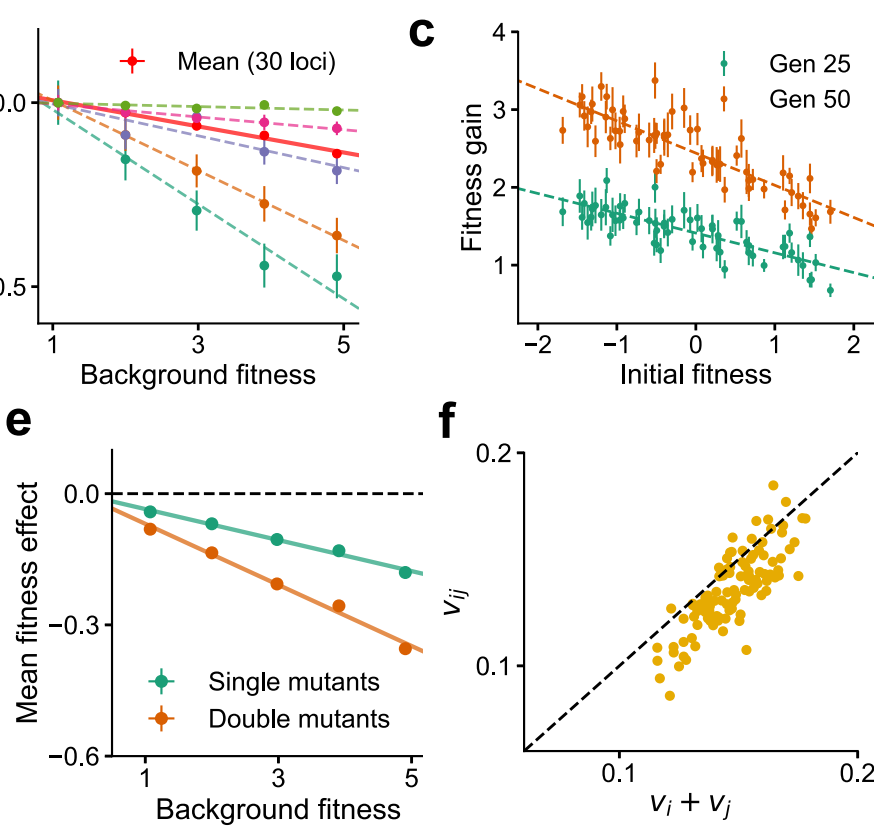

f

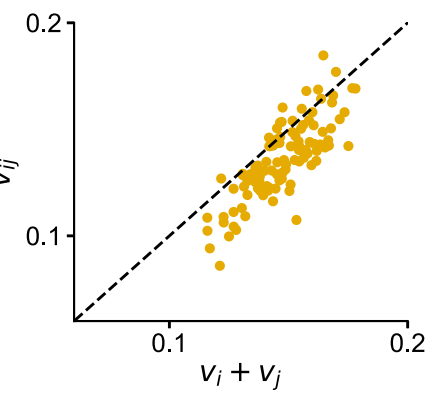

Figure 2. Global epistasis is recapitulated in a generic model of a complex trait and leads to testable predictions. (a) The distribution of variance fractions (DVF) over 400 loci for the simulated genotype-phenotype map. (b) The predicted linear relationship between fitness effect (relative to the fitness effect on the least fit background) and background fitness for the mean over 30 randomly chosen loci (red, solid line) and five loci (dashed lines in colors) is recapitulated. The slope of the linear fit for each locus is proportional to its variance fraction, $v$ (slope $=-2 v$ ). Mean and SE are over backgrounds of approximately equal fitness. See Methods for more details. (c) The mean fitness gain after 25 (green) and 50 (orange) generations of simulated evolution of 768 independently adapting populations with 64 unique founders and 12 replicates each. Means and SEs are computed over the 12 replicates. Error bars are s.e.m. (d) The relationship predicted from theory between the residual variance from the linear fit for each locus and its slope is confirmed in simulations. (e) The mean fitness effect for single mutants at 30 loci and double mutants from all possible pairs of the 30 loci. The slope for the double mutants is predicted to be roughly twice that of single mutants. (f) The estimated variance fraction of a double mutant with mutations at two loci is predicted from theory and confirmed in simulations to be approximately the sum of the variance fractions for single mutations at the two loci. Sub-additivity is due to epistasis between the two loci. See Methods for more details.

Examining the DFE over $\ell$ loci for a randomly chosen genotype of fitness $y$ can be thought of as sampling the fitness effects $s_{1}, s_{2}, \ldots, s_{\ell}$ from the conditional joint distribution $P\left(s_{1}, s_{2}, \ldots, s_{\ell} \mid y\right)$, which generally depends on epistasis. If the number of independent, nonzero epistatic terms is large, then $P\left(s_{1}, s_{2}, \ldots, s_{\ell} \mid y\right)$ is a multivariate normal distribution defined by the means and covariances of the $\ell+1$ variables $y, s_{1}, s_{2}, \ldots, s_{\ell}$, which in turn can be computed in terms of the $f$ 's from Eq. (2). In particular, the conditional means and covariances are $\operatorname{Mean}_{y}\left(s_{i}\right)=-2 v_{i}(y-\bar{y}), \operatorname{Cov}_{y}\left(s_{i}, s_{j}\right)=$ $4 V\left(e_{i j}-v_{i} v_{j}\right)$, where $e_{i j}$ is the epistatic variance fraction between loci $i$ and $j$ and $e_{i i}=v_{i}$. This implies that the conditional correlation between fitness effects is $\left(e_{i j}-v_{i} v_{j}\right) / \sqrt{v_{i} v_{j}\left(1-v_{i}\right)\left(1-v_{j}\right)}$.

The DFE simplifies considerably if we make certain additional assumptions on the magnitude of epistatic interactions. If we assume the typical variance fraction $\bar{v}$ is small (i.e., $\bar{v} \ll 1$ ) and also that $e_{i j}$ is $O\left(\bar{v}^{2}\right)$, then correlations are $O(\bar{v})$ and thus negligible. Then, in a particular sample $s_{1}, s_{2}, \ldots, s_{\ell}$, we can think of each $s_{i}$ as being drawn independently with mean $-2 v_{i}(y-\bar{y})$ and variance $4 v_{i} V$. If $\ell$ is large, this leads to a DFE

$$
\rho(s \mid y)=\int_{0}^{1} d v(2 \sqrt{v V})^{-1} P(v) \varphi\left(\frac{s+2 v(y-\bar{y})}{2 \sqrt{v V}}\right),
$$

where $P(v)$ is the DVF across loci and $\varphi$ is the standard normal pdf. Curiously, the correlations between $s_{i}$ 's vanish when $e_{i j}=v_{i} v_{j}$, in which case the above equation is exact and the DFE is determined entirely by the DVF. Further below, we introduce a specific fitness landscape model for which this relation does hold. Diminishing-returns is naturally incorporated in Eq. (9): the mean of $s$ is $-2 \bar{v}(y-\bar{y})$, i.e., the DFE shifts progressively towards deleterious values with increasing fitness. 

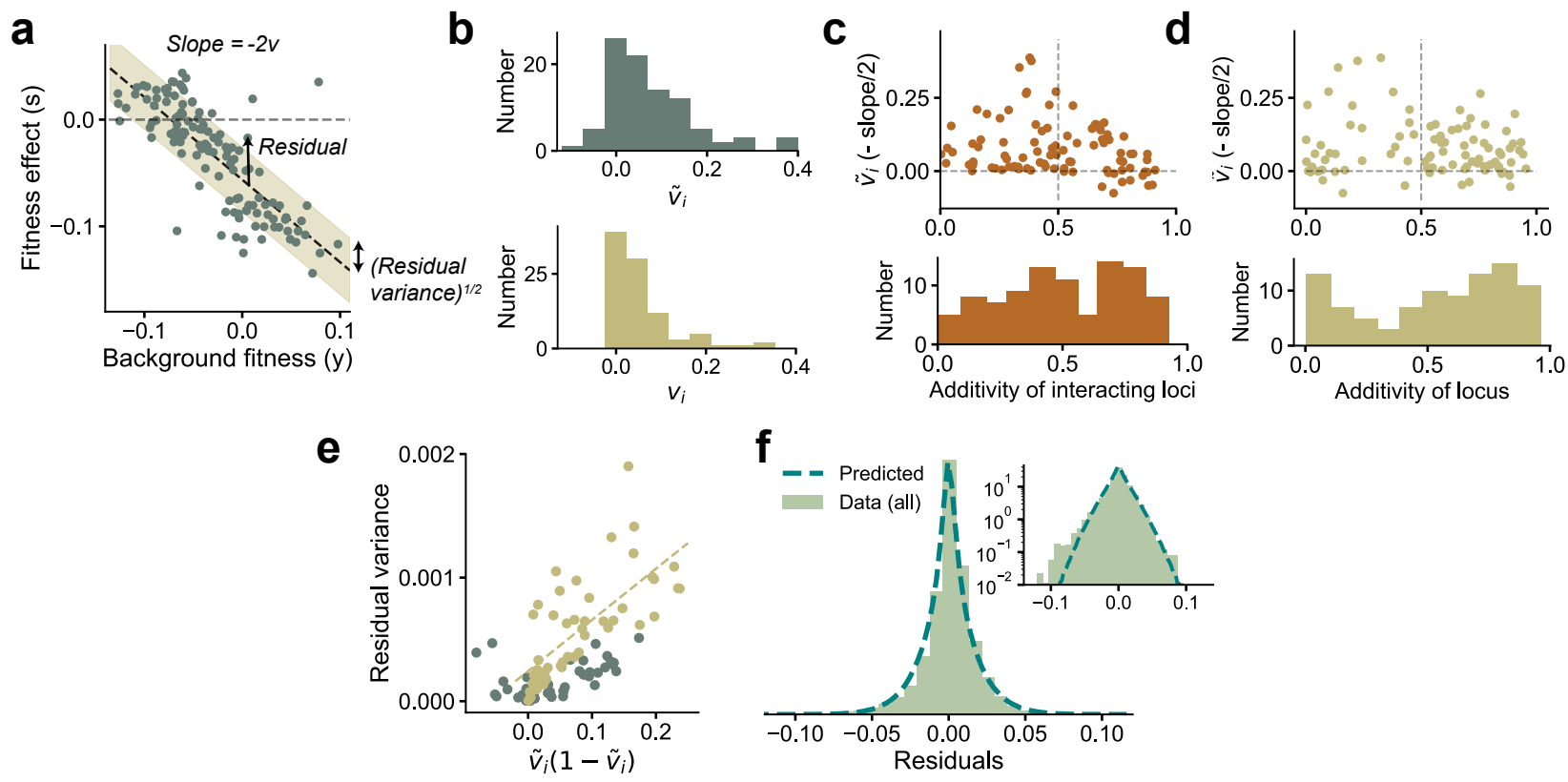

Figure 3. Experimental observations from Johnson et al. 15 are consistent with theoretical predictions. (a) The fitness effect of one of the 91 mutations from [15] plotted against background fitness. (b) The distribution of the measured $\tilde{v}_{i}$ (negative one-half of the slope from (a)) and variance fractions $v_{i}$ for the 91 insertion mutations. (c,d) $\tilde{v}_{i}$ plotted against the additivity of interacting loci and the additivity of the mutated locus (see main text for definitions). The histograms are shown below the plots. The sign of the trend depends on the additivity of interacting loci rather than the additivity of the mutated locus. (e) The measured variance of the residuals against the prediction $\tilde{v}_{i}\left(1-\tilde{v}_{i}\right)$, shown here for the 91 mutations. The yellow circles correspond to the loci with AoIL $<0.5$. The best-fit line (yellow dashed line) to these loci has $R^{2}=0.50\left(R^{2}=0.42\right.$ for all points). (f) The shape of the distribution of residuals pooled from all 91 mutations aligns well with the prediction from Eq. (3). The variances of the two distributions are matched. Inset: same plot in log-linear scale. See Methods for more details.

\section{Historical contingency in adaptive trajectories}

A key unresolved question is the extent to which evolutionary history influences the DFE and the dynamics of adaptation [27]. That is, what does our theory say about historical contingency?

Suppose a clonal population of fitness $y_{0}$ accumulates $k$ successive mutations resulting in fitnesses $y_{1}, y_{2}, \ldots, y_{k}$. By virtue of arising on the same ancestral background, the fitness gain of a new mutation, $s_{k+1}$, is in general correlated with the full sequence of past fitnesses and the identity of the $k$ mutations through its epistatic interactions with them. Based on these correlations, we use well-known properties of conditional normal distributions to write

$$
s_{k+1}=\sum_{i=0}^{k} w_{k+1, i} y_{i}+\epsilon,
$$

where the weights $w_{k+1, i}$ depend on the variance fraction $\left(v_{k+1}\right)$ of the new mutation and its epistatic interactions with past mutations. Here $\epsilon$ is the normallydistributed residual that depends on the initial geno- type and the weights (SI). Eq. (10) is a generalization to a sequence of mutations of Eq. (5), which we can think of as the special case where $k=0$.

To gain intuition, it is useful to first analyze Eq. (10) when $k=1$ (i.e., to compute the effect of a second mutation conditional on the first). In this case, we show in the SI that

$$
s_{2} \simeq-2 v_{2}\left(y_{1}-\bar{y}\right)+\frac{v_{1} v_{2}-e_{12}}{v_{1}} s_{1}+\epsilon,
$$

where $s_{1}=y_{1}-y_{0}$ is the fitness effect due to mutation 1. The first term on the right hand side is the dependence on the fitness of the immediate ancestor, similar to the corresponding term in Eq. (5). The second term quantifies the influence of epistasis between loci 1 and 2 on $s_{2}$. When $e_{12}=v_{1} v_{2}$, dependence on $s_{1}$ vanishes entirely and $s_{2}$ depends only on $y_{1}$. In contrast, if loci 1 and 2 do not interact, $e_{12}=0$, and $s_{2}$ is, on average, larger if the mutation at 1 is beneficial compared to when it is deleterious. This has an intuitive interpretation: diminishing-returns applies to the overall fitness and the mechanism through which it acts is epistasis. However, if mutations 1 and 2 do not interact, then the 
increase in fitness corresponding to mutation 1 does not actually reduce the effect of mutation 2 (as expected by diminishing-returns) so the expected effect of mutation 2 is larger. This analysis suggests that during adaptation, since selection favors mutations with stronger fitness effects on the current background, a mutation that interacts less with previous mutations is more likely to be selected.

To identify the conditions under which history plays a minimal role, we would like to examine when $s_{k+1}$ depends only on the current fitness, $y_{k}$, and is independent of both the past fitnesses and idiosyncratic epistasis. If this were true, then Eq. (5) would apply for new mutations that arise through the course of a single evolutionary path (i.e., the fitness effect of a new mutation is "memoryless" and depends only on its variance fraction and the current fitness). Surprisingly, such a condition does exist. We show that this occurs when the magnitude of epistatic interactions between the new mutation and the $k$ previous mutations, $e_{k+1,1: k}$, satisfies a specific relation: $e_{k+1,1: k}=v_{k+1} v_{1: k}$, where $v_{1: k}$ is the combined variance fraction of the $k$ previous mutations (SI). In general, this condition is not satisfied, implying that there will be historical contingency which can be analyzed using the framework above. Remarkably, it turns out that a fitness landscape model for which the condition is satisfied does exist and arises from certain intuitive assumptions on the organization of biological pathways and cellular processes. This fitness landscape model is described below.

\section{The connectedness model}

We introduce the "connectedness" model (CN model, for short). In this model, each locus $i$ is involved in a fraction $\mu_{i}$ of independent "pathways", where each pathway has epistatic interactions between all loci involved in that pathway (Figure 4a). The probability of an epistatic interaction between three loci $(i, j, k)$ is then proportional to $\mu_{i} \mu_{j} \mu_{k}$, since this is the probability that these loci are involved in the same pathway. When the number of loci $\ell$ is large, we show that in this model, $v_{i}=\mu_{i} /\left(1+\mu_{i}\right)$, and when $\ell$ is small, $v_{i}=\mu_{i} / \bar{\mu} \ell$, where $\bar{\mu}$ is the average over all loci (SI). The CN model therefore has a specific interpretation: the outsized contribution to the fitness from certain loci (large $v_{i}$ ) is due to their involvement in many different complex pathways (large $\mu_{i}$ ) and not from an unusually large perturbative effect on a few pathways. The distribution, $P(\mu)$, across loci determines the DVF.

Statistical fitness landscapes such as the NK model and the Rough Mt. Fuji model [27-32] are related to the CN model. Specifically, the CN model is a sub-class of the broader class of generalized NK models (see 33 for a review). However, often-studied fitness landscape models have one important difference that distinguishes them and gives qualitatively different dynamics of adaptation (shown further below): in contrast to the CN model, classical fitness landscapes are typically 'regular.' That is, the variance fraction of every locus is assumed to be the same (except the star neighborhood model which has a bimodal DVF [33]).

The CN model is equivalent to a Gaussian fitness landscape with exponentially-decaying correlations (SI). The CN model has tunable ruggedness, where the landscape transitions from additivity to maximal epistasis with increasing $\bar{\mu}$. Maximal epistasis corresponds to $\mu_{i}=1$ (and hence $v_{i}=1 / 2$ ) for all $i$. From Eq. (5), this implies that the new fitness after a mutation occurs is independent of the previous fitness, consistent with the expectation from a House-of-Cards model [34] (where genotypes have uncorrelated fitness). Regular fitness landscape models with exponentially-decaying correlations have memoryless fitness effects under the restrictive assumption that every locus is equivalent [27. We show that the dynamics of adaptation of the more general $\mathrm{CN}$ model are also memoryless, i.e., the condition detailed in the previous section holds true (SI). Yet, as we show below, the predicted dynamics for the $\mathrm{CN}$ model are very different to those from a regular fitness landscape model.

We emphasize that the well-connectedness assumed for the CN model is not a requirement for Eq. (5) to hold. However, how diminishing-returns influences the long-term dynamics of adaptation depends on the specific genetic architecture and the corresponding fitness landscape. Consider for example an alternative model of genetic networks organized in a modular structure (Figure 4b). In this model, each locus is part of a single module, and interacts epistatically with other loci in that module to determine the fitness of that module; overall fitness is then determined as a function of the module fitnesses. In this case, the variance contributed by a locus is due to its additive contribution and from epistasis between loci restricted to its module. While the argument for diminishing-returns still applies to the fitness as a whole, it follows from the same argument that diminishing-returns should also apply to each module separately. Consequently, the dynamics of adaptation for the modular model are different from the $\mathrm{CN}$ model. For simplicity, we analyze the dynamics of adaptation for the $\mathrm{CN}$ model and postpone a discussion of how the dynamics differ for different models to subsequent work.

\section{The dynamics of adaptation}

We now examine the DFE that follows from Eq. (5) and what that implies for long-term adaptation under the conditions for memoryless fitness effects. We hence- 
bioRxiv preprint doi: https://doi.org/10.1101/2020.06.14.150946; this version posted November 9, 2020. The copyright holder for this

forth assume a large number of loci with sparse epistasis (though the total number of nonzero epistatic terms is still large). This implies that $\ell \gg 1, v_{i} \ll 1$ and $\bar{v} \ell \gg 1$; we also assume strong-selection-weak-mutation (SSWM) selection dynamics (i.e., a mutation sweeps and fixes in a population before another one arises). It is convenient to rescale fitnesses based on the total variance in fitness across all possible genotypes by defining $z=V^{-1 / 2}(y-\bar{y}), \sigma=V^{-1 / 2} s, \nu=V^{-1 / 2} \eta$. Note that $\nu$ is normally-distributed with zero mean and unit variance. Here $z$ has an intuitive interpretation as the "adaptedness" of the organism. When the organism is neutrally-adapted $(|z| \ll 1)$, positive and negative epistatic contributions to the fitness are balanced and diminishing-returns is negligible. Diminishing-returns is relevant when the organism is well-adapted $(z \gg 1)$. Below, we give the intuition behind our analysis, which is presented in full detail in the SI.

In the neutrally-adapted regime, the linear negative feedback in Eq. (5) is negligible and the DFE is determined by the distribution of $\simeq v^{1 / 2} \nu$. Loci with large $v$ can lead to a DFE with a long tail. If $\bar{v}$ is the typical variance fraction of a locus, the fitness increases as $z \sim n_{s} \bar{v}^{1 / 2}$, where $n_{s}$ is the number of substitutions. Since $\bar{v}$ is a measure of overall epistasis, this implies that epistasis speeds adaptation in the neutrallyadapted regime by allowing access to more influential beneficial mutations.

Fitness increases until the effect of the negative feedback cannot be neglected. From Eq. (5), this happens when $\bar{v} z \sim \bar{v}^{1 / 2} \nu$ (i.e., when $z^{2} \sim \bar{v}^{-1}$ ). Intuitively, fitness begins to plateau when its accumulated benefit from substitutions is comparable to the scale of the total genetic variance $\left(n_{s} \bar{v} \sim 1\right)$ and further improvements are due to rare positive fluctuations. In this welladapted regime, diminishing-returns and increasingcosts epistasis strongly constrain the availability of beneficial mutations, whose effects can be quantified in this model: for a mutation to have a fitness effect $\sigma$, we require from Eq. (5) that $\nu \simeq \sigma / 2 v^{1 / 2}+v^{1 / 2} z$, which has probability $\sim e^{-\nu^{2} / 2}$. Beneficial effects of large $\sigma$ arise when $\nu$ has a large positive deviation. The most likely $v$ that leads to a particular $\sigma$ is when $\nu$ is smallest (i.e., at $v^{*} \simeq \sigma / 2 z$ ), in which case $\nu \simeq \sqrt{2 \sigma z}$, yielding a tail probability $\sim e^{-\sigma z}$. Remarkably, the beneficial DFE in the well-adapted regime is quite generally an exponential distribution independent of the precise form of the DVF (unless it is singular). In particular, we show that for the DFE, $\rho(\sigma \mid z)$,

$$
\frac{\rho(\sigma \mid z)}{\rho(-\sigma \mid z)}=e^{-\sigma z}
$$

which depends solely on the adaptedness of the organism. The exponential form arises because of the Gaussianity of $\nu$, but the argument can be easily extended to $\nu$ with non-Gaussian tails. An exponential beneficial DFE has been previously proposed by Orr [35] but arises here due to a qualitatively different argument (see Discussion).

Under SSWM assumptions, from Eq. (12), the typical effect size of a fixed mutation is $\sigma_{\text {fix }} \sim z^{-1}$, which typically has a variance fraction,

$$
v_{\mathrm{fix}}^{*} \simeq \sigma_{\mathrm{fix}} / 2 z \sim 1 / 2 z^{2} .
$$

The above relation makes precise the effects of increasing-costs epistasis on adaptation. As adaptation proceeds, the delicate balance of high fitness configurations constrains fixed beneficial mutations to have moderate variance fractions. A mutation of small variance fraction is likely to confer small benefit and is lost to genetic drift, while one with a large variance fraction is more likely to disrupt an established high fitness configuration.

This intuition is not captured in regular fitness landscape models, which assume statistically equivalent loci, i.e., $v_{i}=\bar{v}$ for all $i$ and $P(v)=\delta(v-\bar{v})$ is singular. From Eq. (9), we see that this leads to a Gaussian DFE whose mean decreases linearly with increasing fitness, in contrast to the exponential DFE in our theory. The key difference is the lack of loci with intermediate effect, which drive adaptation in the well-adapted regime. As a consequence, the rate of beneficial mutations declines exponentially $\left(U_{b} \sim e^{-\bar{v} z^{2} / 2}\right)$ and the fitness thus sharply plateaus at $z \sim \bar{v}^{-1 / 2}$. In contrast, our theory predicts a much slower depletion of beneficial mutations, $U_{b} \sim z^{-2}(\mathrm{SI})$. The rate of adaptation is $d z / d t \sim U_{b} p_{\text {fix }} \sigma_{\text {fix }} \sim z^{-4}$ (since $p_{\text {fix }} \sim \sigma_{\text {fix }}$ ), which leads to a slow but steady power-law gain in fitness, $z \sim t^{1 / 5}$. The rate of fixation of beneficial mutations is $d n_{s} / d t \sim U_{b} p_{\text {fix }} \sim z^{-3} \sim t^{-3 / 5}$, which gives $n_{s} \sim t^{2 / 5}$.

We verify our analytical results using numerics. As before, we generated a genotype-phenotype map using the CN model with an exponential DVF, $P(v)=$ $\bar{v}^{-1} e^{-v / \bar{v}}$ and $\ell=400$ loci. The DFE in this case can be calculated exactly from Eq. (9):

$$
\rho(\sigma \mid z)=\frac{\bar{v}^{-1}}{2 \sqrt{2 \bar{v}^{-1}+z^{2}}} e^{-\sigma z / 2-|\sigma| \sqrt{2 \bar{v}^{-1}+z^{2}} / 2} .
$$

We simulated the evolution of randomly generated genotypes from $z=0$ to $z=2.5$ and $z=5$ and the DFE across all loci was measured (we chose $\bar{y}=0, V=1$ so that $y=z, s=\sigma)$. The theoretical prediction for the DFE, Eq. (14), closely aligns with the numerical results (Figure 4.).

Due to computational constraints, it is difficult to simulate evolution deep into the well-adapted regime. To compute the shape of adaptive trajectories and their variability, we instead simulated SSWM dynamics using the DFE directly from Eq. (14), beginning from a neutrally-adapted fitness $(z=0)$. Typical trajectories 
bioRxiv preprint doi: https://doi.org/10.1101/2020.06.14.150946; this version posted November 9, 2020. The copyright holder for this
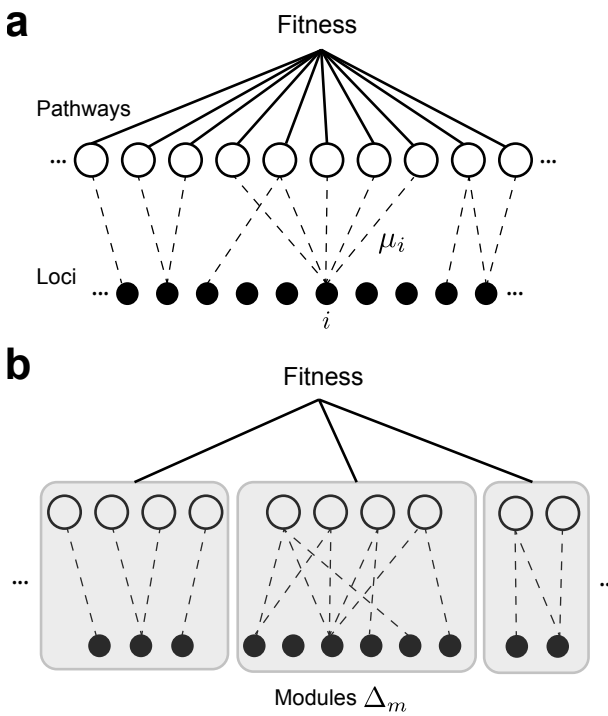
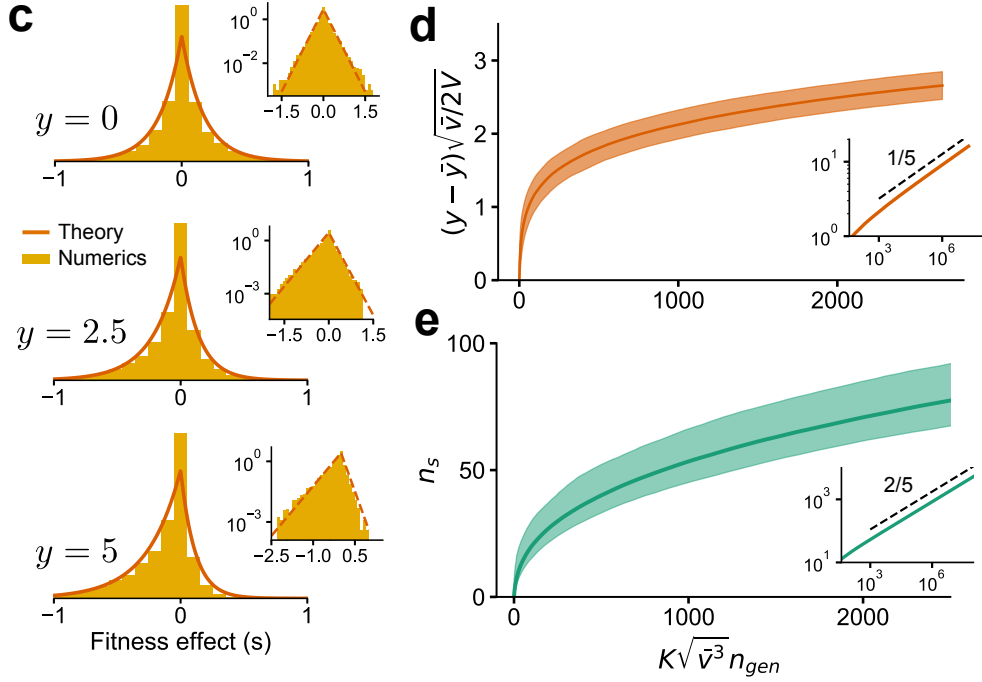

Figure 4. The DFE and long-term adaptation dynamics predicted for the connectedness model. (a) Schematic of the connectedness $(\mathrm{CN})$ model, where each locus is associated with a fraction $\mu$ of pathways that contribute to the organism's fitness. (b) An alternative model with modular organization, where sets of loci interact only within the pathways specific to a single module. (c) The DFE predicted from Eq. (14) matches those obtained from simulated evolution of genotypes from the CN model. 128 randomly drawn genotypes (400 loci) with initial fitness $y$ close to zero are evolved to $y=2.5$ and $y=5$ and the DFE is measured across loci and genotypes. We chose $\bar{y}=0$ and $V=1$ so that $y$ represents adaptedness. Insets: same plots in log-linear scale. Note that the number of beneficial mutations acquired during the simulated evolution $(\sim 10-20)$ is much less than the total number of loci (400). (d) For a neutrally-adapted organism, the theory predicts quick adaptation to a well-adapted state beyond which the adaptation dynamics are independent of the specific details of the genotype-fitness map. Shown here is the mean adaptation curve predicted under strong-selection-weak-mutation (SSWM) assumptions, which leads to a power-law growth of fitness with exponent $1 / 5$ in the well-adapted regime (inset). (e) The number of fixed beneficial mutations under SSWM, which grows as a power-law with exponent $2 / 5$ in the well-adapted regime (inset). The shaded region is the $95 \%$ confidence interval around the mean for (c) and (d). See Methods and SI for more details.

(Figure 4 $\mathrm{d}$ ) show rapid adaptation to the well-adapted regime beyond which the fitness grows slowly as $t^{1 / 5}$, as predicted from theory. The predictions for the number of fixed beneficial mutation are also re-capitulated (Figure 4).

\section{DISCUSSION}

Recent empirical studies have observed consistent patterns of diminishing-returns and increasing-costs epistasis. Our model gives a simple mechanistic explanation for these observations. In particular, we showed that these patterns are generic consequences of widespread microscopic epistatic interactions. The intuition underlying this result is that a random mutation typically has a larger disruptive effect on the delicate balance of microscopic epistasis that underpins a fitter background. Our model predicts a quantitative relationship between the magnitudes of global epistasis (i.e., the negative slope of diminishing-returns and increasing-costs epistasis) and microscopic epista- sis, which we confirmed using existing data (Figure 3).

A similar explanation for diminishing-returns and increasing-costs epistasis has been recently proposed by Lyons et al. [16]. While our core argument for diminishing-returns and increasing-costs epistasis is the same as in that work, our Fourier analysis framework dissects the features of the fitness landscape necessary to observe these phenomena in terms of experimentally measurable average effects (i.e., the f's in Eq. (2)). In particular, we show that the additivity of a locus' interacting partners critically determines whether the trend is negative or unbiased. In addition, the Fourier analysis framework yields predictions for the distribution of fitness effects, the historical influence of past mutations on the fitness effect of a newly mutated site and motivates the proposed 'connectedness' fitness landscape model. The analysis of experimental data presented in Lyons et al. complements the experimental data considered here, lending further empirical support for the prevalence of epistasis and its importance in determining long-term adaptability.

Our model leads to other experimentally testable pre- 
bioRxiv preprint doi: https://doi.org/10.1101/2020.06.14.150946; this version posted November 9,2020 . The copyright holder for this

dictions. The most direct and accessible test of the theory is to measure the fitness for all possible combinations of mutations at $\sim 10-15$ significant loci and compare (using Eq. (6)) the magnitude of global epistasis to the measured fitness coefficients (the $f$ 's). Additionally, we predict that the magnitude of global epistasis of a double mutant should be nearly the sum of magnitudes of the corresponding single mutants, and any deviations should be biased towards sub-additivity. Since the predictions involve measuring residual variance, experimental noise can be an important confounding factor.

We emphasize that our key result, Eq. (5), is a general statistical relation that holds irrespective of the specific genetic architecture and the corresponding fitness landscape. Weak epistasis with many loci is sufficient to observe noticeable patterns of global epistasis. However, the argument fails if the contribution of a locus is purely additive or when epistasis is limited to one or a handful of other loci. In the latter case, we expect the fitness effect of a mutation to be dominated by the allelic states of its partner loci, and thus take on a few discrete values. A few examples from Johnson et al. [15] indeed exhibit this pattern, (e.g. cases where the fitness effect of a specific mutation depends primarily on the allelic state at a single other locus).

We highlight a distinction between global epistasis discussed in this work and another form of global epistasis (also known as "unidimensional" or "nonspecific" epistasis) typically used in protein evolution to describe nonspecific epistatic interactions due to a nearly additive trait transformed by a nonlinear function 23 25. 36, 37]. This nonlinear function creates systematic relationships between epistasis terms and breaks the condition of independent epistatic terms required for our arguments to apply. Specific nonlinearities such as an exponential function may indeed lead to a negative linear trend on average, but the structure of the residuals differs from the one in Eq. (5) and observed in data.

A surprising empirical observation is that the negative linear relationship between fitness effect and ancestral fitness characteristic of global epistasis has different slopes for different loci. Our model identifies the negative slope as twice the fraction of variance contributed by a locus to the trait. To explain the wide range of variance fractions (VF) observed in data, we developed the connectedness $(\mathrm{CN})$ model, a framework to think about the organization of cellular processes that can lead to loci of widely varying VFs. In the $\mathrm{CN}$ model, loci have a large VF due to their involvement in many different pathways rather than due to a large effect on a single pathway. The $\mathrm{CN}$ model can be viewed as a statistical fitness landscape where loci can have a range of VFs, specified by the distribution of variance fractions (DVF). In the special case of every locus having the same VF, the $\mathrm{CN}$ model corresponds to a fitness landscape with tunable ruggedness and exponentiallydecaying correlations.

Extending our framework to incorporate adaptation, we showed that the distribution of fitness effects (DFE) depends only on the current fitness, rather than the entire evolutionary history, under the intuitive assumptions behind the CN model. The theory therefore gives a simple explanation for why phenotypic evolution can be predictable, even while the specific mutations that underlie this evolution are highly stochastic.

Our framework has an implicit notion of 'adaptedness' without referencing a Gaussian-shaped phenotypic optimum, often assumed in models of adaptation (e.g. Fisher's geometric model) 38-40. Over the course of adaptation, the DFE shifts towards deleterious values, reflecting diminishing-returns, which naturally arises from our basic arguments. For a well-adapted organism, we show that the DFE for beneficial mutations takes on an exponential form, and leads to universal adaptive dynamics. While an exponential DFE for beneficial mutations has been proposed previously based on extreme value theory [35], our result arises due to an entirely different argument: the tail of the beneficial DFE is determined by loci of intermediate size whose disruptive effect due to increasing-costs is small, yet whose effect size is large enough not to be lost due to genetic drift. Our theory further predicts declining adaptability, with rapid adaptation in a neutrally-adapted regime followed by much slower increases in fitness, resulting in power-law adaptive trajectories when the organism is well-adapted. This is consistent with observations from the E.coli LTEE [1, 2]. Our model predicts a quicker decline in the number of substitutions $\left(n_{s} \sim t^{2 / 5}\right)$ compared to the near linear trend observed in the LTEE data 4; however, the dynamics of fixation in the LTEE deviate strongly from SSWM assumptions, which may explain the discrepancy.

\section{METHODS AND MATERIALS}

The code and data to generate the figures are available at 41].

\section{Simulations}

We use a fitness landscape model with $\ell$ loci to generate the genotype-fitness map. Each locus is assigned a sparsity $\mu$ from $P(\mu)$, which is an exponential distribution with mean $\bar{\mu}$. Each of $M$ independent pathways sample loci with each locus $i$ having probability $\mu_{i}$ of being selected to a pathway. We choose $\ell=400, \bar{\mu}=0.02, M=500$ so that $\bar{\mu} \ell=8$ ensures 
bioRxiv preprint doi: https://doi.org/10.1101/2020.06.14.150946; this version posted November 9, 2020. The copyright holder for this preprint (which was not certified by peer review) is the author/funder, who has granted bioRxiv a license to display the preprint in perpetuity. It is made available under aCC-BY-NC 4.0 International license.

signficant epistasis. All loci in a pathway interact with each other, where additive and higher-order coefficient terms of all orders were drawn independently from a standard normal distribution. The total fitness is the sum of contributions from the $M$ pathways. We normalize the coefficients so that the sum of squares of all coefficients is 1 , i.e., the total variance across genotypes is 1 . The mean, $\bar{y}$ is close to zero from our sampling procedure. The above procedure is a simple and efficient way to generate epistatic terms to order $\sim 20$, beyond which the computational requirements are limited by the exponentially increasing demand. Note that the effects described in the paper were also observed with only pairwise and cubic epistatic terms.

The variance fractions shown in Figure $2 a$ can be calculated numerically from the definition. From the theory, given our choice of $P(\mu)$, these should follow an exponential distribution with mean $\bar{v} \approx \bar{\mu} /(1+\bar{\mu})$. There may be deviations since $M$ is finite whereas the calculations assume $M \rightarrow \infty$. To generate Figure $2 \mathrm{~b}$, in order to get a range of background fitnesses, we first sample 128 random genotypes. These have fitnesses close to zero; in order to obtain a range of fitness values, we simulated the evolution of these 128 genotypes up to $y=1,2,3,4,5$ under strong-selection-weak-mutation (SSWM) assumptions to get $128 \times 5=640$ genotypes at roughly five fitness values. The fitness effect of applying a mutation (i.e., flipping its sign) is measured for 30 randomly chosen loci (which are kept fixed) over each of the 640 genotypes. This is shown for five of the 30 and for the mean over the 30 loci in Figure $2 \mathrm{~b}$.

To generate Figure 2r, we sampled 64 random genotypes and 12 replicates of each. The evolution of these 768 genotypes was simulated for a total of 50 generations with a mutation rate of 1 per generation. The mean fitness gain over the 12 replicates is plotted for each of the 64 founders against their initial fitness.

To generate Figure 2 $\mathrm{d}$, the residuals are measured using the same procedure as for the experimental data analysis described below for the initial 128 genotypes at $y \approx 0$ and the 30 loci with the largest variance fraction.

Double mutants were created by mutating all pairs of the 30 randomly chosen loci on the 640 evolved genotypes. Their mean fitness effect was computed and plotted along with the mean fitness effect for single mutants, shown in Figure 2. The variance fraction of the pair of loci for the double mutant was estimated as before and compared to the sum of the estimated variance fractions of the corresponding single mutants. This is shown in Figure 2:

To generate the plots in Figure 4k, we simulated the evolution of 128 randomly sampled genotypes to $y=2.5$ and $y=5$. The fitness effect of 200 randomly sampled loci was measured and the distribution is plotted.

\section{Analysis of the data from Johnson et al.}

The data from Johnson et al. 15 consists of the fitness after the addition of 91 insertion mutations on each of 145 background genotypes. The fitness of a particular mutation at locus $i$ can be modeled as

$$
y_{i}=-c_{i} y+b_{i}+\operatorname{Residual}_{i}(g),
$$

where $y_{i}, y$ are the mutant and background fitnesses respectively, $c_{i}, b_{i}$ are constants for each locus and the residual $\operatorname{Residual}_{i}(g)$ depends on the background genotype $g$.

We estimate the variance fraction $v_{i}=\left(1-\hat{\rho}_{i}\right) / 2$, where the Pearson correlation $\hat{\rho}_{i}=\operatorname{Corr}\left(y_{i}, y\right)$ across backgrounds, while $\tilde{v}_{i}$ is estimated as the negative onehalf of the slope of the best linear fit of $s_{i}$ and $y$. The residuals for each of the 145 genotypes for each of the 91 mutations is simply

$$
\operatorname{Residual}_{i}(g)=\left(y_{i}+c_{i} y\right)-\overline{\left(y_{i}+c_{i} y\right)},
$$

where the overline represents an average over the 145 genotypes, which is used as an estimate of the constant term and $c_{i}$ is either $2 v_{i}-1$ or $2 \tilde{v}_{i}-1$. In Figure $3 \mathrm{p}$, we plot the distribution of estimated $v_{i}$ and $\tilde{v}_{i}$. In Figure $3 \mathrm{c}$, we compute the AoIL for each locus using Eq. (7), which we show in the SI to be $\left|\operatorname{Cov}\left(s_{i}, y_{i}+y\right)\right| /\left(\left|\operatorname{Cov}\left(s_{i}, y_{i}+y\right)\right|+\operatorname{Var}\left(s_{i}\right)\right)$. In Figure $3 \mathrm{~d}$, we compute the additivity using Eq. (8). The additive effect is $f_{i}=\overline{\left(y_{i}-y\right)} / 2$ and $\operatorname{Var}\left(s_{i}\right) / 4$ gives the sum of squares of the epistatic terms (SI). In Figure 3e, we compute the variance of the residuals across the 145 genotypes for each locus and plot it against the locus' estimated $\tilde{v}_{i}\left(1-\tilde{v}_{i}\right)$. In Figure 3 ; , we plot the distribution of residuals over all genotypes and loci. The prediction is that in the WE limit the distribution of residuals is determined by $2 \tilde{v}_{i}\left(1-\tilde{v}_{i}\right) \eta$, where $\eta$ is a Gaussian random variable. We multiply $\sqrt{\tilde{v}_{i}\left(1-\tilde{v}_{i}\right)}$ for each locus with 10,000 i.i.d standard normal RVs, pool the resulting numbers for all loci and plot the predicted distribution in Figure 3: The distributions are variancematched. While Figure 3e shows that the variance of the residuals aligns with the theoretical prediction of being proportional to slope, Figure 3f shows that the data is also consistent with the predicted Gaussianity of the background-genotype-dependent contribution.

\section{ACKNOWLEDGMENTS}

We thank Sergey Kryazhimskiy, Andrew Murray, Milo Johnson, and members of the Desai lab for comments on the manuscript. G.R was supported by the NSF-Simons Center for Mathematical and Statistical Analysis of Biology at Harvard (award number 
bioRxiv preprint doi: https://doi.org/10.1101/2020.06.14.150946; this version posted November 9,2020 . The copyright holder for this

\#1764269) and the Harvard FAS Quantitative Biology Initiative. M.M.D. acknowledges support from the
Simons Foundation (Grant 376196), NSF Grant PHY1914916, and NIH grant R01GM104239.
[1] Wiser MJ, Ribeck N, Lenski RE. Long-term dynamics of adaptation in asexual populations. Science. 2013;342(6164):1364-1367.

[2] Lenski RE, Wiser MJ, Ribeck N, Blount ZD, Nahum JR, Morris JJ, et al. Sustained fitness gains and variability in fitness trajectories in the long-term evolution experiment with Escherichia coli. Proceedings of the Royal Society B: Biological Sciences. 2015;282(1821):20152292.

[3] Tenaillon O, Barrick JE, Ribeck N, Deatherage DE, Blanchard JL, Dasgupta A, et al. Tempo and mode of genome evolution in a 50,000-generation experiment. Nature. 2016;536(7615):165-170.

[4] Good BH, McDonald MJ, Barrick JE, Lenski RE, Desai MM. The dynamics of molecular evolution over 60,000 generations. Nature. 2017;551(7678):45-50.

[5] Kryazhimskiy S, Rice DP, Jerison ER, Desai MM. Global epistasis makes adaptation predictable despite sequence-level stochasticity. Science. 2014;344(6191):1519-1522.

[6] Elena SF, Lenski RE. Evolution experiments with microorganisms: the dynamics and genetic bases of adaptation. Nature Reviews Genetics. 2003;4(6):457-469.

[7] Perfeito L, Sousa A, Bataillon T, Gordo I. Rates of fitness decline and rebound suggest pervasive epistasis. Evolution. 2014;68(1):150-162.

[8] Wünsche A, Dinh DM, Satterwhite RS, Arenas CD, Stoebel DM, Cooper TF. Diminishing-returns epistasis decreases adaptability along an evolutionary trajectory. Nature Ecology \& Evolution. 2017;1(4):1-6.

[9] Sanjuán R, Cuevas JM, Moya A, Elena SF. Epistasis and the adaptability of an RNA virus. Genetics. 2005;170(3):1001-1008.

[10] Couce A, Tenaillon OA. The rule of declining adaptability in microbial evolution experiments. Frontiers in genetics. 2015;6:99.

[11] Jerison ER, Kryazhimskiy S, Mitchell JK, Bloom JS, Kruglyak L, Desai MM. Genetic variation in adaptability and pleiotropy in budding yeast. Elife. 2017;6:e27167.

[12] Schenk MF, Szendro IG, Salverda ML, Krug J, De Visser JAG. Patterns of epistasis between beneficial mutations in an antibiotic resistance gene. Molecular biology and evolution. 2013;30(8):1779-1787.

[13] Khan AI, Dinh DM, Schneider D, Lenski RE, Cooper TF. Negative epistasis between beneficial mutations in an evolving bacterial population. Science. 2011;332(6034):1193-1196.

[14] Chou HH, Chiu HC, Delaney NF, Segrè D, Marx CJ. Diminishing returns epistasis among beneficial mutations decelerates adaptation. Science. 2011;332(6034):1190-1192.

[15] Johnson MS, Martsul A, Kryazhimskiy S, Desai MM. Higher-fitness yeast genotypes are less robust to deleterious mutations. Science. 2019;366(6464):490-493.
[16] Lyons DM, Zou Z, Xu H, Zhang J. Idiosyncratic epistasis creates universals in mutational effects and evolutionary trajectories. Nature Ecology \& Evolution. 2020;p. 1-9.

[17] Hordijk W, Stadler PF. Amplitude spectra of fitness landscapes. Advances in Complex Systems. 1998;1(01):39-66

[18] Neher RA, Shraiman BI. Statistical genetics and evolution of quantitative traits. Reviews of Modern Physics. 2011;83(4):1283.

[19] Weinberger ED. Fourier and Taylor series on fitness landscapes. Biological cybernetics. 1991;65(5):321-330.

[20] Szendro IG, Schenk MF, Franke J, Krug J, De Visser JAG. Quantitative analyses of empirical fitness landscapes. Journal of Statistical Mechanics: Theory and Experiment. 2013;2013(01):P01005.

[21] Weinreich DM, Lan Y, Wylie CS, Heckendorn RB. Should evolutionary geneticists worry about higherorder epistasis? Current opinion in genetics \& development. 2013;23(6):700-707.

[22] Poelwijk FJ, Krishna V, Ranganathan R. The contextdependence of mutations: a linkage of formalisms. PLoS computational biology. 2016;12(6):e1004771

[23] Starr TN, Thornton JW. Epistasis in protein evolution. Protein Science. 2016;25(7):1204-1218.

[24] Sailer ZR, Harms MJ. Detecting high-order epistasis in nonlinear genotype-phenotype maps. Genetics. 2017;205(3):1079-1088.

[25] Otwinowski J, McCandlish DM, Plotkin JB. Inferring the shape of global epistasis. Proceedings of the National Academy of Sciences. 2018;115(32):E7550 E7558.

[26] Bloom JS, Kotenko I, Sadhu MJ, Treusch S, Albert FW, Kruglyak L. Genetic interactions contribute less than additive effects to quantitative trait variation in yeast. Nature communications. 2015;6(1):1-6.

[27] Agarwala A, Fisher DS. Adaptive walks on highdimensional fitness landscapes and seascapes with distance-dependent statistics. Theoretical population biology. 2019;130:13-49.

[28] Neidhart J, Szendro IG, Krug J. Exact results for amplitude spectra of fitness landscapes. Journal of theoretical biology. 2013;332:218-227.

[29] Kauffman SA, Weinberger ED. The NK model of rugged fitness landscapes and its application to maturation of the immune response. Journal of theoretical biology. 1989;141(2):211-245.

[30] Stadler PF, Happel R. Random field models for fitness landscapes. Journal of Mathematical Biology. 1999;38(5):435-478.

[31] Aita T, Uchiyama H, Inaoka T, Nakajima M, Kokubo $\mathrm{T}$, Husimi Y. Analysis of a local fitness landscape with a model of the rough Mt. Fuji-type landscape: Application to prolyl endopeptidase and thermolysin. Biopolymers: Original Research on Biomolecules. 
$2000 ; 54(1): 64-79$.

[32] Altenberg L. Back T, Foger DB, Michalewicz Z, editors. NK Fitness Landscapes, In "The Handbook of Evolutionary Computation". Oxford Univ. Press; 1997.

[33] Hwang S, Schmiegelt B, Ferretti L, Krug J. Universality classes of interaction structures for NK fitness landscapes. Journal of Statistical Physics. 2018;172(1):226278.

[34] Kauffman S, Levin S. Towards a general theory of adaptive walks on rugged landscapes. Journal of theoretical Biology. 1987;128(1):11-45.

[35] Orr HA. The distribution of fitness effects among beneficial mutations. Genetics. 2003;163(4):1519-1526.

[36] Otwinowski J. Biophysical inference of epistasis and the effects of mutations on protein stability and function. Molecular biology and evolution. 2018;35(10):23452354.

[37] Husain K, Murugan A. Physical Constraints on Epistasis. Molecular Biology and Evolution. 2020 05; Cvaa128.

[38] Fisher R. The genetical theory of natural selection. Clarendon Press; 1930.

[39] Orr HA. The genetic theory of adaptation: a brief history. Nature Reviews Genetics. 2005;6(2):119-127.

[40] Martin G, Lenormand T. A general multivariate extension of Fisher's geometrical model and the distribution of mutation fitness effects across species. Evolution. 2006;60(5):893-907.

[41] Reddy G. github:greddy992/global_epistasis; 2020. Available from: https://github.com/greddy992/ global_epistasis 


\section{CONTENTS}

2 I. Diminishing-returns and increasing-costs epistasis in a model of a complex trait

A. Fourier representation of the fitness function 


\section{I. DIMINISHING-RETURNS AND INCREASING-COSTS EPISTASIS IN A MODEL ${ }_{25}$ OF A COMPLEX TRAIT}

In the main text, we propose that the fitness effect after a mutation at locus $i$ can be written 27 as

$$
s_{i}=-2 v_{i}(y-\bar{y})+2 \sqrt{v_{i}\left(1-v_{i}\right)} \eta_{i}, \quad 0 \leq v_{i} \leq 1 .
$$

${ }_{28}$ Here $\eta_{i}$ is a genotype and locus-dependent contribution, which is distributed as a mean-zero ${ }_{29}$ Gaussian with variance equal to the total genetic variance, $\bar{y}$ is a constant, and $v_{i}$ is the variance 30 fraction defined further below. Eq. (11) corresponds to the symmetric case where the fitness effects 31 of the mutations $x_{i}=-1 \rightarrow+1$ and $x_{i}=+1 \rightarrow-1$ are simultaneously regressed against their 32 respective background fitness. The directed case when the mutation is specified to change from ${ }_{33} x_{i}=-1 \rightarrow+1$ (or $x_{i}=+1 \rightarrow-1$ ) will be considered in Section IC

We will now show that Eq. (1) arises under certain conditions in a generic model of a complex 35 trait with $\ell \gg 1$ biallelic loci. Essentially, we would like to compute the distribution of the new 36 fitness, $y_{i}$, across genotypes after a mutation at locus $i$ given the current fitness $y$ and the set of all 37 parameters $\Theta$ of the model (i.e. $P\left(y_{i} \mid y, \Theta\right)$, with $s_{i}=y_{i}-y$ ). Using the chain rule of probability, 38 we can write

$$
P\left(y_{i} \mid y, \Theta\right)=\sum_{g} P\left(y_{i} \mid g, \Theta\right) P(g \mid y, \Theta)
$$

39 where the sum is over all possible genotypes. While $P\left(y_{i} \mid g, \Theta\right)$ is determined by the genotype40 fitness map, $P(g \mid y, \Theta)$ is the crucial factor that gives weight only to the genotypes that yield the 41 current fitness $y$. If the fitness is much larger than the mean fitness over all possible genotypes, ${ }_{42}$ Eq. (2) implicitly ensures that weight is given to only those genotypic configurations that lead to 43 such an unusually large fitness. We will analyze the case of a "microcanonical ensemble" where 44 every genotypic configuration that leads to a particular fitness is equally likely (with no linkage) 45 i.e., the prior $P(g)$ across genotypes is uniform (see Section ID 2 for a discussion).

46 It is difficult to directly evaluate the sum in Eq. (2). In the following sections, we give a 47 simple derivation but elaborated to highlight the key assumption that leads to Eq. (1). In short, ${ }_{48}$ the negative correlation between $s_{i}=y_{i}-y$ and $y$ implied by Eq. (1) is a trivial consequence ${ }_{49}$ of $y_{i}$ and $y$ having the same distribution w.r.t $P(g) . \eta_{i}$ is in general arbitrary and determined ${ }_{50}$ by the genotype-fitness map. However, $\eta_{i}$ is normally-distributed if we make certain assumptions 
51 about the structure of epistasis in the genotype-fitness map. The emergence of the negative linear 52 relationship for a directed mutation $x_{i}=-1 \rightarrow+1$ is subtler.

\section{A. Fourier representation of the fitness function}

54 The fitness $y(g)$ for a genotype of length $\ell, g=\left\{x_{1}, x_{2}, \ldots, x_{\ell}\right\}$, where $x_{i}= \pm 1$, can be 55 generally written as

$$
y(g)=\bar{y}+\sum_{i} f_{i} x_{i}+\sum_{i>j} f_{i j} x_{i} x_{j}+\sum_{i>j>k} f_{i j k} x_{i} x_{j} x_{k}+\ldots
$$

${ }_{56}$ The symmetric choice of $x_{i}= \pm 1$ is chosen for mathematical convenience. In this form, the total 57 variance contribution from the $q$ th order epistatic terms is $\sum_{i_{1}>i_{2}>\ldots i_{q}} f_{i_{1} i_{2} \ldots i_{q}}^{2}$ and the total genetic 58 variance, defined as

$$
V \equiv \frac{1}{2^{\ell}} \sum_{g}(y(g)-\bar{y})^{2},
$$

59 is the sum of variance contributions from orders $q=1$ to $\ell$, i.e., the sum of squares of all the $f$ 's. ${ }_{60}$ The sum over the $2^{\ell}$ genotypes is an expectation value assuming all genotypes are equally likely; 61 we will denote this expectation using an overline hereafter. We use this expectation value as a 62 proxy for empirical averages over the "background" genotypes in a population. With a sufficient 63 number of background genotypes, the empirical average should converge to this expectation value. 64 The representation in Eq. (3) is a Fourier representation of the fitness function on the $\ell$ 65 dimensional hypercube, and makes calculations much simpler. For instance, to get the terms ${ }_{66}$ from each order, we have

$$
\bar{y}=\overline{y(g)}, \quad f_{i}=\overline{x_{i} y(g)}, \quad f_{i j}=\overline{x_{i} x_{j} y(g)}, \quad \ldots
$$

${ }_{67}$ It is useful to define the variance contribution due to a particular locus $i$ as (symmetry w.r.t ${ }_{68}$ interchanging indices is used for each term throughout)

$$
V_{i} \equiv f_{i}^{2}+\sum_{j \neq i} f_{i j}^{2}+\sum_{k<j \neq i} f_{i j k}^{2}+\ldots
$$

${ }_{69}$ We also define the variance fraction of locus $i$,

$$
v_{i} \equiv \frac{V_{i}}{V},
$$

70 which plays a key role in the model. 


\section{B. Derivation of Eq. (1)}

We would like to relate the fitness before and after locus $i$ is flipped (i.e. $x_{i} \rightarrow-x_{i}$ ), denoted by $y$ and $y_{i}$ respectively. We have from Eq. (3),

$$
\begin{aligned}
y-\bar{y} & =y_{i}+\xi_{i}, \\
y_{i}-\bar{y} & =y_{\bar{i}}-\xi_{i},
\end{aligned}
$$

72 where $y_{\bar{i}}$ and $\xi_{i}$ respectively contain all the terms not containing and containing locus $i$, i.e.,

$$
\xi_{i}=f_{i} x_{i}+\sum_{j \neq i} f_{i j} x_{i} x_{j}+\sum_{k<j \neq i} f_{i j k} x_{i} x_{j} x_{k}+\ldots
$$

We have $y_{\bar{i}}=\left(y+y_{i}\right) / 2-\bar{y}$ and $\xi_{i}=\left(y-y_{i}\right) / 2$. Since $y_{\bar{i}}$ and $\xi_{i}$ contain all the genotype-dependence, we can write

$$
\begin{aligned}
P\left(y_{i} \mid y, \Theta\right) & \propto P\left(y_{i}, y \mid \Theta\right) \\
& =\int P\left(y_{i}, y \mid y_{\bar{i}}, \xi_{i}\right) P\left(y_{\bar{i}}, \xi_{i} \mid \Theta\right) d y_{i} \xi_{i} \\
& =P\left(y_{\bar{i}}=\frac{y+y_{i}}{2}-\bar{y}, \xi_{i}=\frac{y-y_{i}}{2} \mid \Theta\right) .
\end{aligned}
$$

${ }_{73}$ From the properties of the Fourier representation in Eq. (5), it is easy to see that the means are ${ }_{74} \overline{y_{\bar{i}}}=0, \overline{\xi_{i}}=0$, the variances are $\overline{y_{\bar{i}}^{2}}=V-V_{i}, \overline{\xi_{i}^{2}}=V_{i}$, and the covariance is $\overline{y_{\bar{i}} \xi_{i}}=0$.

75 The calculations so far have been exact. We now make the key assumption that $y_{\bar{i}}$ and $\xi_{i}$ are 76 both normal-distributed across genotypes. This assumption is similar to that of Fisher's infinites77 imal model, where the distribution of trait values across strains for a complex trait is argued 78 to be normal-distributed since the trait value is due to infinitesimal independent contributions 79 from many loci. While $y_{\bar{i}}$ is easily seen to be normal-distributed for large $\ell$, an argument can be so made for $\xi_{i}$ only if locus $i$ has a large number of independent, nonzero epistatic terms and the 81 additive term $f_{i}$ is smaller in magnitude than the epistatic terms; specifically, we require that ${ }_{82} f_{i}^{2} \lesssim \sum_{j \neq i} f_{i j}^{2}+\sum_{k<j \neq i} f_{i j k}^{2}+\ldots$ If instead $f_{i}^{2} \gg \sum_{j \neq i} f_{i j}^{2}+\sum_{k<j \neq i} f_{i j k}^{2}+\ldots$, then $\xi_{i}$ is bi-modal, 83 where the two modes correspond to $\xi_{i} \approx \pm f_{i}$ at $x_{i}= \pm 1$. For loci with pairwise and third-order ${ }_{84}$ epistasis, the number of pairwise and third-order epistatic terms scale $\propto \ell$ and $\propto \ell^{2}$ respectively, ${ }_{85}$ which justifies the normality assumption for large $\ell$ even if individual epistatic terms are smaller 86 in magnitude than the additive terms. 
${ }_{87}$ Under these assumptions and since $y_{\bar{i}}$ and $\xi_{i}$ are linearly independent, we have

$$
P\left(y_{i} \mid y, \Theta\right) \propto \varphi\left(\frac{y+y_{i}-2 \bar{y}}{2 \sqrt{V-V_{i}}}\right) \varphi\left(\frac{y-y_{i}}{2 \sqrt{V_{i}}}\right)
$$

88 where $\varphi$ is the standard normal pdf. Therefore, $y_{i}$ is normal-distributed across genotypes and 89 from the above equation can be written as

$$
y_{i}-\bar{y}=\left(1-2 v_{i}\right)(y-\bar{y})+2 \sqrt{v_{i}\left(1-v_{i}\right)} \eta_{i}
$$

9o where the variance fraction $v_{i}=V_{i} / V$ was defined previously and $\overline{\eta_{i}}=0, \overline{\eta_{i}^{2}}=V$. This leads to 91 the form in Eq. (1),

$$
s_{i}=-2 v_{i}(y-\bar{y})+2 \sqrt{v_{i}\left(1-v_{i}\right)} \eta_{i} .
$$

92 The above derivation was presented to clarify the basic assumptions. Simply computing the 93 covariance between $y$ and $y_{i}$ in Eq. (8) and Eq. (9), we get $\overline{(y-\bar{y})\left(y_{i}-\bar{y}\right)}=\overline{y_{\bar{i}}^{2}}-\overline{\xi_{i}^{2}}=V-2 V_{i}$. ${ }_{94}$ The correlation is then $1-2 v_{i}$. Eq. (16) follows if additionally $y_{i}, y$ are jointly Gaussian, which 95 is true if locus $i$ has many independent, nonzero epistatic terms.

\section{Directed mutation}

97 Previously, we considered the symmetric flip $x_{i} \rightarrow-x_{i}$ and averaged over all $\ell$ loci including $i$. ${ }_{98}$ Here, we consider the case when the mutation is specified to change either from $x_{i}=-1 \rightarrow+1$ 99 or $x_{i}=+1 \rightarrow-1$. In this case, we should average over all loci except $i$.

We consider $x_{i}=-1 \rightarrow+1$ (the opposite case is similar). The fitness before the mutation is

$$
\tilde{y}_{i}=\bar{y}-f_{i}+\sum_{j \neq i}\left(f_{j}-f_{i j}\right) x_{j}+\sum_{j>k \neq i}\left(f_{j k}-f_{i j k}\right) x_{j} x_{k}+\ldots,
$$

101 and the fitness after the mutation is

$$
\hat{y}_{i}=\bar{y}+f_{i}+\sum_{j \neq i}\left(f_{j}+f_{i j}\right) x_{j}+\sum_{j>k \neq i}\left(f_{j k}+f_{i j k}\right) x_{j} x_{k}+\ldots,
$$

102 so that

$$
s_{i}=\hat{y}_{i}-\tilde{y}_{i}=2 f_{i}+2 \sum_{j \neq i} f_{i j} x_{j}+2 \sum_{j>k \neq i} f_{i j k} x_{j} x_{k}+\ldots
$$


The tilde and hat are used here to distinguish the fitness from the $y_{i}$ defined previously for the symmetric case corresponding to the flip $x_{i} \rightarrow-x_{i}$. Using orthogonality relations, we have for the means, variance and covariance,

$$
\begin{aligned}
\bar{s}_{i} & =2 f_{i} \\
\overline{\tilde{y}_{i}} & =\bar{y}-f_{i}, \\
\overline{\left(s_{i}-\bar{s}_{i}\right)^{2}} & =4 \sum_{j \neq i} f_{i j}^{2}+4 \sum_{j>k \neq i} f_{i j k}^{2}+\ldots \\
\overline{\left(\tilde{y}_{i}-\overline{\tilde{y}}_{i}\right)^{2}} & =\sum_{j \neq i}\left(f_{j}-f_{i j}\right)^{2}+\sum_{j>k \neq i}\left(f_{j k}-f_{i j k}\right)^{2}+\ldots \\
\overline{\left(s_{i}-\bar{s}_{i}\right)\left(\tilde{y}_{i}-\overline{\tilde{y}}_{i}\right)} & =2 \sum_{j \neq i}\left(f_{j}-f_{i j}\right) f_{i j}+2 \sum_{j>k \neq i}\left(f_{j k}-f_{i j k}\right) f_{i j k}+\ldots \\
& =2\left(\sum_{j \neq i} f_{j} f_{i j}+\sum_{j>k \neq i} f_{j k} f_{i j k}+\ldots\right)-\frac{\left(s_{i}-\bar{s}_{i}\right)^{2}}{2}
\end{aligned}
$$

The slope when $s_{i}$ is regressed against $\tilde{y}_{i}$ is $\overline{\left(s_{i}-\bar{s}_{i}\right)\left(\tilde{y}_{i}-\overline{\tilde{y}}_{i}\right)} / \overline{\left(\tilde{y}_{i}-\overline{\tilde{y}}_{i}\right)^{2}}$. We can define a "modified" variance fraction $\tilde{v}_{i}$ as half the negative-slope,

$$
\begin{aligned}
\tilde{v}_{i} & \equiv \frac{\overline{\left(s_{i}-\bar{s}_{i}\right)^{2}} / 4-\left(\sum_{j \neq i} f_{j} f_{i j}+\sum_{j>k \neq i} f_{j k} f_{i j k}+\ldots\right)}{\sum_{j \neq i}\left(f_{j}-f_{i j}\right)^{2}+\sum_{j>k \neq i}\left(f_{j k}-f_{i j k}\right)^{2}+\ldots}, \\
& =\frac{\left(\sum_{j \neq i} f_{i j}^{2}+\sum_{j>k \neq i} f_{i j k}^{2}+\ldots\right)-\left(\sum_{j \neq i} f_{j} f_{i j}+\sum_{j>k \neq i} f_{j k} f_{i j k}+\ldots\right)}{\sum_{j \neq i}\left(f_{j}-f_{i j}\right)^{2}+\sum_{j>k \neq i}\left(f_{j k}-f_{i j k}\right)^{2}+\ldots} .
\end{aligned}
$$

${ }_{103}$ Writing the linear form based on this correlation, we get

$$
s_{i}=2 f_{i}-2 \tilde{v}_{i}\left(\tilde{y}_{i}-\bar{y}+f_{i}\right)+K_{i} \eta_{i}
$$

104 where $\eta_{i}$ is again normally distributed (in the widespread epistasis (WE) limit) with zero mean 105 and variance $\overline{\left(\tilde{y}_{i}-\overline{\tilde{y}}_{i}\right)^{2}}$ and $K_{i}^{2}=\frac{\overline{\left(s_{i}-\bar{s}_{i}\right)^{2}}}{\left(\tilde{y}_{i}-\tilde{\tilde{y}}_{i}\right)^{2}}-4 \tilde{v}_{i}^{2}$. Note that, unlike $v_{i}, \tilde{v}_{i}$ can be negative.

However, we argue that $\tilde{v}_{i}$ is typically positive in the WE limit, which leads to a negative linear 107 trend. The second term in the numerator on the right hand side of Eq. (26) has the same number 108 of terms as $\overline{\left(s_{i}-\bar{s}_{i}\right)^{2}}$ but these terms appear as products of Fourier coefficients that may have 109 opposing signs. In particular, if $\sum_{j \neq i} f_{i j}^{2}>\sum_{j \neq i} f_{j} f_{i j}, \sum_{j>k \neq i} f_{i j k}^{2}>\sum_{j \neq i} f_{j k} f_{i j k}$ and so on, then ${ }_{110} \tilde{v}_{i}$ is guaranteed to be positive. If we denote the typical magnitude of $q$ th order epistasis terms as ${ }_{111} e_{q}$ ( $e_{1}$ corresponds to additive effects), each of this relationships has the form $e_{q+1}^{2} \ell>e_{q} e_{q+1} \sqrt{\ell}$ 
112 when $\ell \gg 1$, i.e., $e_{q}<e_{q+1} \sqrt{\ell}$. If the number of loci is sufficiently large, then these relationships 113 will hold even if the typical magnitude of individual higher-order epistasis terms are smaller than 114 the lower-order terms. We therefore expect that the second term in the numerator on the right 115 hand side of Eq. 26 is smaller than $\frac{\overline{\left(s_{i}-\bar{s}_{i}\right)^{2}}}{2}$ when $\ell \gg 1$. A similar argument can be made for 116 the cross terms $f_{j} f_{i j}, f_{j k} f_{i j k}, \ldots$ once the squares in the denominator of the right hand side of ${ }_{117}$ Eq. (26) are expanded.

${ }_{118}$ When $\ell \gg 1$, we can then write

$$
\tilde{v}_{i} \approx \frac{V_{i}-f_{i}^{2}}{V-f_{i}^{2}}=\frac{v_{i}-f_{i}^{2} / V}{1-f_{i}^{2} / V} \approx v_{i}-f_{i}^{2} / V
$$

${ }_{119}$ Further, in the WE limit, $K_{i}^{2} \approx 4 \tilde{v}_{i}-4 \tilde{v}_{i}^{2}$ so that the variance of $K_{i} \eta_{i}$ is $\propto \tilde{v}_{i}\left(1-\tilde{v}_{i}\right)$.

120 To estimate the ratio of the magnitudes of the second and first terms in the numerator on the 121 right hand side of Eq. (26) from data, we use the expression for the covariance,

$$
\overline{\left(s_{i}-\bar{s}_{i}\right)\left(\hat{y}_{i}-\overline{\hat{y}}_{i}\right)}=2 \sum_{j \neq i}\left(f_{j}+f_{i j}\right) f_{i j}+2 \sum_{j>k \neq i}\left(f_{j k}+f_{i j k}\right) f_{i j k}+\ldots,
$$

122 to get

$$
\frac{\left|\sum_{j \neq i} f_{j} f_{i j}+\sum_{j>k \neq i} f_{j k} f_{i j k}+\ldots\right|}{\sum_{j \neq i} f_{i j}^{2}+\sum_{j>k \neq i} f_{i j k}^{2}+\ldots}=\frac{\left|\operatorname{Cov}\left(s_{i}, \tilde{y}_{i}\right)+\operatorname{Cov}\left(s_{i}, \hat{y}_{i}\right)\right|}{\operatorname{Var}\left(s_{i}\right)}=\frac{\left|\operatorname{Cov}\left(s_{i}, \tilde{y}_{i}+\hat{y}_{i}\right)\right|}{\operatorname{Var}\left(s_{i}\right)}
$$

\section{${ }_{123}$ D. Comments on the result}

The negative linear trend observed in data may arise due to the measured fitness being a non126 linear function of an unobserved additive trait. In this case, the epistasis terms are systematically ${ }_{127}$ related to each other and the independence assumptions used to derive Eq. (16) break down. ${ }_{128}$ In short, we show that specific nonlinearities can indeed lead to a negative linear trend, but the 129 statistics of the residuals observed in data make this possibility unlikely.

130 Suppose the fitness is $y=\phi(u)$, where $\phi$ is a nonlinear function, $u=f_{0}+\sum_{i} f_{i} x_{i}$ is the 131 unobserved additive trait, $f_{0}$ is a constant and $f_{i}$ are additive coefficients. For a linear trend, we 132 require $s_{i}=\phi\left(u_{i}\right)-\phi(u) \propto \phi(u)$, where $u_{i}=u-2 f_{i} x_{i}$ for the flip $x_{i} \rightarrow-x_{i}$. For small $f_{i}$ relative 133 to the other coefficients, we can Taylor expand $\phi\left(u_{i}\right)$ and show that we require $\phi(u) \propto e^{u}$ to get a 134 linear trend. This nonlinearity creates a linear trend with slope $e^{-2 f_{i} x_{i}}-1$. For a negative linear 
135 trend, we require $2 f_{i} x_{i}>0$. However, even if this condition is true, the relation $s_{i}=\left(e^{-2 f_{i} x_{i}}-1\right) y$ 136 is exact and there are no residuals.

137 To introduce residuals, suppose instead that $u=f_{0}+\sum_{i} f_{i} x_{i}+h$, where $h$ is a House-of-Cards $138(\mathrm{HoC})$ term, i.e., it is an independent Gaussian random variable (mean 0, variance $\sigma_{h}^{2}$ ) across 139 genotypes and repeatable across measurements. $h$ introduces epistasis in the unobserved trait. ${ }_{140}$ We have $s_{i}=\left(e^{-2 f_{i} x_{i}+h_{i}-h}-1\right) y$, where $h_{i}$ is the HoC term after the mutation, so that the average 141 fitness effect conditional on $y$ is $\bar{s}_{i}=\left(e^{-2 f_{i} x_{i}+\sigma_{h}^{2}}-1\right) y$. The conditional variance of the residuals 142 is $\overline{\left(s_{i}-\bar{s}_{i}\right)^{2}}=e^{-4 f_{i} x_{i}+2 \sigma_{h}^{2}}\left(e^{2 \sigma_{h}^{2}}-1\right) y^{2}$. Note that the residual variance is no longer proportional 143 to the slope and this variance increases as $y^{2}$, which are both inconsistent with the data.

\section{Maximum entropy interpretation}

The expectation values are averages over all the genotypes assuming that every genotype of a 146 particular fitness is equally likely i.e., the distribution over genotypes is uniform. This assumption 147 is analogous to ensemble averages over a microcanonical ensemble in statistical physics, where one 148 assumes that all the particle configurations that have a particular energy are equally likely. The ${ }_{149}$ experimental setting in Johnson et al [1] is similar. The background genotypes are generated from 150 a cross between two strains, which due to recombination makes each locus have equal probability 151 of being one of the two alleles. Closely-linked loci may be considered together as blocks. Some of 152 the loci are partially linked, which may lead to deviations from the predictions. The expressions 153 derived above can be easily extended to the case with different background genotype statistics. 154 The uniform distribution has an information-theoretic interpretation as the distribution that 155 has the maximum entropy (MaxEnt) given no additional knowledge of how the genotype was 156 generated. Eq. (16) can therefore be viewed as the MaxEnt prediction of the fitness effect if 157 locus $i$ is mutated conditioned on the current observed fitness $y$. A key idea that will be used 158 throughout the paper is that when each locus $i$ has a significant number of independent, nonzero 159 epistatic terms, the distribution of fitness and fitness effects is jointly normal with respect to the 160 uniform prior over genotypes. From well-known properties of multivariate normal distributions, 161 the MaxEnt predictions of unobserved variables are multilinear forms of the observed variables. ${ }_{162}$ For example, the MaxEnt prediction for $s_{i}$ given an observed sequence of past fitness is an 163 autoregressive Gaussian process defined by the covariance between the unobserved and observed 
164 variables (Section III B).

\section{Varying a subset of loci}

In addition, the sums in Eq. (3) are over $\ell$ loci involved in a trait. In reality, we may vary a subset $P$ of all loci and take averages over only this subset. The derivation still follows through in this case; we can simply write the fitness in terms of effective parameters as

$$
\begin{gathered}
y(g)=\tilde{y}+\sum_{i \in P} \tilde{f}_{i} x_{i}+\sum_{i, j \in P} \tilde{f}_{i j} x_{i} x_{j}+\ldots, \quad \text { where } \\
\tilde{y}=\bar{y}+\sum_{i \notin P} f_{i} x_{i}+\sum_{i, j \notin P} f_{i j} x_{i} x_{j}+\ldots, \\
\tilde{f}_{i}=f_{i}+\sum_{j \notin P} f_{i j} x_{j}+\sum_{j, k \notin P} f_{i j k} x_{j} x_{k}+\ldots, \quad \text { and so on. }
\end{gathered}
$$

Here we are abusing notation - it is to be assumed that a coefficient with a particular combination of indices appears only once in the sums. The results from the previous section still apply w.r.t these new effective parameters. For instance, the total variance and the variance due to locus $i \in P$ are

$$
\begin{aligned}
\tilde{V} & =\sum_{i \in P} \tilde{f}_{i}^{2}+\sum_{i, j \in P} \tilde{f}_{i j}^{2}+\sum_{i, j, k \in P} \tilde{f}_{i j k}^{2}+\ldots, \\
\tilde{V}_{i} & =\tilde{f}_{i}^{2}+\sum_{j \in P} \tilde{f}_{i j}^{2}+\sum_{j, k \in P} \tilde{f}_{i j k}^{2}+\ldots,
\end{aligned}
$$

166 and the effective variance fraction is $\tilde{V}_{i} / \tilde{V}$.

\section{Relationship to the fluctuation-dissipation relation}

Eq. (16) is analogous to the fluctuation-dissipation theorem in statistical physics, which re169 lates the response of a thermodynamic system to a perturbation. The relationship between the 170 magnitude of macroscopic epistasis (the slope in Eq. 16) and the variance due to the back171 ground genotypes is analogous to the relationship between viscous drag and Brownian motion. 172 For Brownian motion, the normality arises due to numerous, independent collisions of a particle 173 with neighboring particles. In our case, natural selection acts as an external perturbation which 174 pushes the system away from equilibrium (here $\bar{y}$ ). Diminishing-returns naturally arises as the 175 tendency of the system to revert to its entropically-favored equilibrium state. 
Using the arguments from the previous section, it is easy to show that the variance of a double mutant at loci $i$ and $j, V_{i j}$, is necessarily sub-additive. In particular, we have

$$
\begin{aligned}
V_{i j} & =V_{i}+V_{j}-2 I_{i j}, \quad \text { where } \\
I_{i j} & =f_{i j}^{2}+\sum_{k \neq i, j} f_{i j k}^{2}+\ldots
\end{aligned}
$$

177 is the total epistatic variance between loci $i$ and $j$. The correlation between the new fitness after 178 a double mutation and the previous fitness is $1-2 V_{i j} / V \equiv 1-2 v_{i j}=1-2 v_{i}-2 v_{j}+2 e_{i j}$, where ${ }_{179} e_{i j} \equiv I_{i j} / V$ is the epistatic variance fraction between $i$ and $j$.

\section{II. CONNECTEDNESS MODEL}

We introduce a "connectedness" model (the CN model, for short), where each locus has a 182 probability $\mu_{i}$ of being involved in any particular interaction. We can interpret $\mu_{i}$ as the fraction of 183 independent pathways that involve locus $i$, where each pathway has epistatic interactions between ${ }_{184}$ all loci involved in that pathway. The number of independent pathways, $M$, is assumed to be 185 very large. The probability of an epistatic interaction between, say loci $i, j, k$, is proportional 186 to $\mu_{i} \mu_{j} \mu_{k}$, since this is the probability that these loci are involved in the same pathway. The 187 magnitude of the interaction term is $f_{i j k}^{2} \propto \mu_{i} \mu_{j} \mu_{k}$, where the proportionality is the magnitude 188 of the perturbative effect of the mutations, which is assumed to be constant for all orders of 189 interaction. We set this quantity to unity since it simply scales the fitness coefficients and does 190 not affect subsequent results. The $\mathrm{CN}$ model leads to a specific interpretation (and hence its 191 name): the outsized contribution to the variance from a particular locus is due to its involvement 192 in many different complex pathways and not from an unusually large perturbative effect on a 193 few pathways. For large $\ell$, the $\mathrm{CN}$ model is specified by the distribution, $P(\mu)$, across loci. In 194 particular, given $P(\mu)$, we can calculate the total genetic variance, $V$, and the variance due to 195 locus $i, V_{i}$. We define $\bar{\mu} \equiv \int_{0}^{1} \mu P(\mu) d \mu$.

We calculate the expected total variance across statistical ensembles. Note that here the expectations are averages over ensembles where the parameters of the model are re-sampled, in

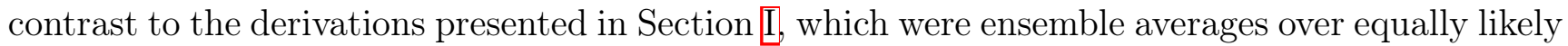


genotypes. Since each pathway is independently sampled, expectations approximate the values in a single realization as $M \rightarrow \infty$. All expectations are denoted $\langle$.$\rangle . We calculate the expected$ variance contribution from one pathway: since all pathways are statistically identical, the total variance from $M$ pathways is simply $M$ times the expected contribution from a single pathway. The contribution from the $q$ th order interaction between loci $i_{1}, i_{2}, \ldots, i_{q}$ is $\left\langle f_{i_{1} i_{2} \ldots i_{q}}^{2}\right\rangle=\prod_{n=1}^{q} \mu_{i_{n}}$. The expected total variance is

$$
\begin{aligned}
V & =\sum_{i}\left\langle f_{i}^{2}\right\rangle+\sum_{i>j}\left\langle f_{i j}^{2}\right\rangle+\sum_{i>j>k}\left\langle f_{i j k}^{2}\right\rangle+\ldots, \\
& =\sum_{i} \mu_{i}+\sum_{i>j} \mu_{i} \mu_{j}+\sum_{i>j>k} \mu_{i} \mu_{j} \mu_{k}+\ldots, \\
& =\prod_{i=1}^{\ell}\left(1+\mu_{i}\right)-1
\end{aligned}
$$

The variance due to terms involving locus $i$ is

$$
\begin{aligned}
V_{i} & =\left\langle f_{i}^{2}\right\rangle+\sum_{j \neq i}\left\langle f_{i j}^{2}\right\rangle+\sum_{j>k \neq i}\left\langle f_{i j k}^{2}\right\rangle+\ldots, \\
& =\mu_{i}+\mu_{i} \sum_{j \neq i} \mu_{j}+\mu_{i} \sum_{j>k \neq i} \mu_{j} \mu_{k}+\ldots, \\
& =\mu_{i} \prod_{j \neq i}\left(1+\mu_{j}\right) .
\end{aligned}
$$

196 Therefore, we have

$$
v_{i}=V_{i} / V=\mu_{i} \frac{\prod_{j \neq i}\left(1+\mu_{j}\right)}{\prod_{j}\left(1+\mu_{j}\right)-1} .
$$

${ }_{197}$ There are two qualitatively different regimes, $\bar{\mu} \ell \ll 1$ and $\bar{\mu} \ell \gg 1$. When $\bar{\mu} \ell \ll 1$, each pathway 198 typically contains a single locus and should lead to an additive model. In this limit, we can write 199 from Eq. (45),

$$
v_{i} \approx \frac{\mu_{i}}{\ell \bar{\mu}}, \quad \bar{\mu} \ell \ll 1
$$

200 which is consistent with the expectation that $\sum_{i} v_{i}=1$ and $v_{i} \sim \ell^{-1}$ for an additive model. In 201 the opposite limit, $\bar{\mu} \ell \gg 1$, we have

$$
v_{i} \approx \frac{\mu_{i}}{1+\mu_{i}}, \quad \bar{\mu} \ell \gg 1
$$

${ }_{202}$ The CN model therefore leads to an intuitive interpretation of the variance fraction as being 203 determined by the sparsity of interactions of a locus. Eq. (47) yields an upper bound at $v_{i}=0.5$, 
204 at $\mu_{i}=1$. From Eq. (15), we see that this case is equivalent to the House-of-Cards model of 205 maximal epistasis where the new fitness after a mutation is independent of the previous fitness. 206 The DVF $P(v)$ is directly determined by $P(\mu)$; the CN model can therefore be used as a generative 207 model to generate fitness landscapes with arbitrary DVFs.

We can further calculate the epistatic variance between two loci in the CN model. The total epistatic variance $I_{i j}$ between loci $i$ and $j$ is

$$
\begin{aligned}
I_{i j} & =\left\langle f_{i j}^{2}\right\rangle+\sum_{k \neq i, j}\left\langle f_{i j k}^{2}\right\rangle+\ldots \\
& =\mu_{i} \mu_{j} \prod_{k \neq i, j}\left(1+\mu_{k}\right) .
\end{aligned}
$$

208 In the limit $\bar{\mu} \ell \gg 1$, the epistatic variance fraction after dividing by $V$ is then simply $e_{i j}=$ $209 \mu_{i} \mu_{j} /\left(1+\mu_{i}\right)\left(1+\mu_{j}\right)=v_{i} v_{j}$. Using Eq. (37), we have

$$
v_{i j}=v_{i}+v_{j}-2 v_{i} v_{j} .
$$

${ }_{210}$ If $v_{i}$ 's are small, the $\mathrm{CN}$ model predicts near-additivity between the effects of two loci. This is not 211 inconsistent with the strong epistasis assumption implicit in the limit $\bar{\mu} \ell \gg 1$ : though the total 212 contribution of epistatic interactions to the genetic variance may be large, the epistatic variance 213 between two specific loci can still be negligible. This is because the majority of epistatic variance 214 is due to the combinatorially large number of higher-order epistatic terms whose individual effects 215 themselves can be weak.

\section{A. Relationship to statistical fitness landscapes}

217 Statistical fitness landscapes such as the NK model and the Rough Mt. Fuji model are closely ${ }_{218}$ related to the $\mathrm{CN}$ model described above. The $\mathrm{CN}$ model falls under the broad class of generalized ${ }_{219}$ NK models [2]. In generalized NK models, epistasis is due to modules (or "pathways") of $K$ loci 220 that interact epistatically with each other. The different NK models differ in how the loci are 221 assigned to the modules and the interaction structure within the module. In the CN model, each 222 locus has a locus-specific probability of being part of any module and the interaction structure 223 within a module is all-to-all. The locus-specific probability gives rise to a highly non-regular 224 model, i.e., loci can have a wide range of contributions to the total variance. This feature gives 
225 rise to qualitatively different adaptation properties. We will show this further below in addition 226 to showing that the $\mathrm{CN}$ model has a special memoryless property.

${ }_{227}$ Well-studied fitness landscapes such as Kauffman's NK model and the Rough Mt. Fuji land228 scape are regular i.e., every locus contributes equally to the variance. In other words, the DFE, 229 which is determined by the DVF in our picture instead comes from a single constant value $v=\bar{v}$. ${ }_{230}$ The fluctuations of the fitness effects are solely due to the genotype-dependent term $\eta$, which is 231 a Gaussian. In Section IIIA, we show that the DFE in this case corresponds to a Gaussian with 232 mean $-2 \bar{v}(y-\bar{y})$. As adaptation proceeds and $y$ increases, the DFE shifts to the deleterious 233 side but retains its Gaussian shape. The adaptation properties that result from this DFE are ${ }_{234}$ quite different from those arising from our theory. Further, while the regular fitness landscape 235 picture may lead to a good approximation of our results when the number of mutations is large, 236 it does not capture the different magnitudes of diminishing-returns for different loci observed in 237 experiments.

\section{B. The landscape of the regular CN model}

The CN model for the special case of homogeneous loci, $\mu_{i}=\bar{\mu}$ for all $i$, is similar to the NK 240 model but with one major difference: the number of loci in each pathway in the NK model is ${ }_{241}$ fixed at $K$ loci, whereas in the $\mathrm{CN}$ model the typical pathway size is controlled by a continuous 242 parameter $\bar{\mu}$. This introduces contributions at every epistatic order while effectively imposing 243 sparsity on the contributions from higher-order interactions. We now show that the regular CN 244 model has tunable ruggedness, i.e., it transitions from an additive model to a model with maximal 245 epistasis with increasing $\bar{\mu}$ and has exponentially-decaying correlations for $\bar{\mu} \ell \gg 1$.

The regular fitness landscapes often discussed are stationary Gaussian processes on a $\ell$ dimensional hypercube. The regular CN model (i.e., $\mu_{i}=\bar{\mu}$ for all $i$ ) also falls into this class as $M \rightarrow \infty$. The key quantity that defines such a fitness landscape is the covariance function between the fitnesses of genotypes $g$ and $g^{\prime}, C\left(g, g^{\prime}\right)=C(d)$, where $d \equiv\left|g-g^{\prime}\right|$ is the Hamming distance between two genotypes. We now compute the covariance $C(d)$ for the regular $\mathrm{CN}$ model. Each order term in each pathway is independent and the covariances for each order add up over all pathways. We first calculate the expectation of the first order term in a single pathway, which 
is

$$
\begin{aligned}
\sum_{i, i^{\prime}}\left\langle f_{i} x_{i} f_{i^{\prime}} x_{i^{\prime}}^{\prime}\right\rangle & =\bar{\mu} \sum_{i, i^{\prime}} \delta_{i i^{\prime}} x_{i} x_{i^{\prime}}^{\prime} \\
& =\bar{\mu} g \cdot g^{\prime} \\
& =\bar{\mu}(\ell-2 d) .
\end{aligned}
$$

Here we have used $\left\langle f_{i}^{2}\right\rangle=\bar{\mu}$, where the $\bar{\mu}$ comes from the probability of locus $i$ being selected for the module as argued previously. The covariance is linear in the distance between genotypes, as one would expect from an additive model. Directly calculating the higher order terms is more complicated because of the ordering restriction $i>j$ for the second order term and for higher orders. As noted previously [3] [5], these can instead be calculated using combinatorics, which we will demonstrate with the second-order term. We have for the second-order covariance

$$
\begin{aligned}
\sum_{i>j, i^{\prime}>j^{\prime}}\left\langle f_{i j} x_{i} x_{j} f_{i^{\prime} j^{\prime}} x_{i^{\prime}}^{\prime} x_{j^{\prime}}^{\prime}\right\rangle & =\bar{\mu}^{2} \sum_{i>j, i^{\prime}>j^{\prime}} \delta_{i i^{\prime}} \delta_{j j^{\prime}} x_{i} x_{i^{\prime}}^{\prime} x_{j} x_{j^{\prime}}^{\prime} \\
& =\bar{\mu}^{2} \sum_{i>j}\left(g g^{\prime}\right)_{i}\left(g g^{\prime}\right)_{j},
\end{aligned}
$$

246 where the element-wise product $\left(g g^{\prime}\right)_{i} \equiv x_{i} x_{i}^{\prime}$ is 1 if $x_{i}=x_{i}^{\prime}$ match and -1 otherwise. If $d$ is the ${ }_{247}$ distance between $g$ and $g^{\prime}$ then the element-wise product $g g^{\prime}$ has $d-1$ terms and $\ell-d 1$ terms. ${ }_{248}$ The term in the summation above is 1 if both $\left(g g^{\prime}\right)_{i}$ and $\left(g g^{\prime}\right)_{j}$ are chosen from the $\ell-d$ subset or 249 both are chosen from $d$ subset. This term is -1 if one of the two is chosen from the $\ell-d$ subset 250 and the other from the $d$ subset. The number of terms that are 1s are therefore $\left(\begin{array}{c}\ell-d \\ 2\end{array}\right)\left(\begin{array}{c}d \\ 0\end{array}\right)+\left(\begin{array}{c}\ell-d \\ 0\end{array}\right)\left(\begin{array}{c}d \\ 2\end{array}\right)$ 251 and the number of terms that are -1 s are $\left(\begin{array}{c}\ell-d \\ 1\end{array}\right)\left(\begin{array}{l}d \\ 1\end{array}\right)$. This argument is easily extended to higher ${ }_{252}$ orders. The general $q$ th order contribution to the covariance is

$$
\bar{\mu}^{q} \sum_{k=0}^{\min (q, d)}(-1)^{k}\left(\begin{array}{l}
\ell-d \\
q-k
\end{array}\right)\left(\begin{array}{l}
d \\
k
\end{array}\right) .
$$

${ }_{253}$ It is easily verified that the first-order term matches. When $d=0$, we recover the binomial coef${ }_{254}$ ficients, as expected. The summation above is precisely the Krawtchouk polynomial $\mathcal{K}_{q}(d ; \ell, 2)$, 255 which we will denote by $\mathcal{K}_{q}(\ell, d)$. We therefore have

$$
C(d)=\sum_{q=1}^{d} \bar{\mu}^{q} \mathcal{K}_{q}(\ell, d) .
$$


256 The generating function of the Krawtchouk polynomials yields

$$
C(d)=(1+\bar{\mu})^{\ell-d}(1-\bar{\mu})^{d}-1 .
$$

${ }_{257}$ The above expression is consistent with intuition: when $\bar{\mu} \ell \ll 1$, the covariance is linear in $d$, as 258 expected for an additive model. In the opposite limit of $\bar{\mu} \ell \gg 1$ and $0<d<\ell / 2$, the constant 259 term 1 can be ignored, and we see that the covariance is proportional to $e^{-\lambda d}$, where $\lambda=\ln \left(\frac{1+\bar{\mu}}{1-\bar{\mu}}\right)$. 260 Epistasis is maximal when $\bar{\mu} \rightarrow 1$, in which case $\lambda \rightarrow \infty$ and the covariance rapidly goes to zero 261 with $d$. This is the House-of-Cards model of maximal epistasis.

\section{The landscape of the general CN model}

${ }_{263}$ The landscape of the general CN model, where loci can have different $\mu_{i}$, is no longer stationary 264 but the correlation structure can still be calculated. The $q$ th order contribution to the covariance 265 between genotypes $g$ and $g^{\prime}$ in the general case is

$$
\sum_{i_{1}>i_{2}>\cdots>i_{q}}\left\langle f_{i_{1} i_{2} \ldots i_{q}}^{2}\right\rangle \prod_{n=1}^{q}\left(x_{i_{n}} x_{i_{n}}^{\prime}\right)=\sum_{i_{1}>i_{2}>\cdots>i_{q}} \prod_{n=1}^{q} \mu_{i_{n}} x_{i_{n}} x_{i_{n}}^{\prime} .
$$

${ }_{266}$ It is easy to see that when the contributions from all orders are added up, the covariance $C\left(g, g^{\prime}\right)$ 267 has a rather simple product form

$$
C\left(g, g^{\prime}\right)=\prod_{i=1}^{\ell}\left(1+\mu_{i} x_{i} x_{i}^{\prime}\right)-1
$$

268 The correlation $c\left(g, g^{\prime}\right)=C\left(g, g^{\prime}\right) / \sqrt{C(g, g) C\left(g^{\prime}, g^{\prime}\right)}$ is

$$
c\left(g, g^{\prime}\right)=\frac{\prod_{i=1}^{\ell}\left(1+\mu_{i} x_{i} x_{i}^{\prime}\right)-1}{\prod_{i=1}^{\ell}\left(1+\mu_{i}\right)-1} .
$$

${ }_{269}$ The above relation is exact. When $\mu_{i} \ll 1, \bar{\mu} \ell \gg 1$, then $1 \pm \mu_{i} \approx e^{ \pm \mu_{i}}$ and the 1 's in the 270 numerator and denominator above can be ignored. We get

$$
c\left(g, g^{\prime}\right) \approx e^{-2 \sum_{i: x_{i} \neq x_{i}^{\prime}} \mu_{i}}
$$

${ }_{271}$ When $\mu_{i}$ 's are equal we recover the homogeneous case with exponentially-decaying correlations. 
272 III. ADAPTATION 275 stochastic dynamical processes that lead to fixation. Before analyzing properties of adaptive 276 walks, we clarify what our previous analysis, which ultimately led to Eq. (16), means for the 277 DFE.

278 279 over all genotypes in the population that have fitness $y$. We are instead interested in the DFE, 280 where fitness effects are measured for all the mutations of a particular genotype that has fitness $281 y$. The DFE for a particular genotype generally depends on idiosyncratic epistatic interactions 282 between loci. In order to make this explicit, we turn to our analysis framework from Section [1. ${ }_{283}$ For notational convenience, we will assume $\bar{y}=0$ and $V=1$ in this sub-section and the next.

284 The DFE over $\ell$ loci for a particular genotype of a fitness $y$ can be thought of as a sample, $285 s_{1}, s_{2}, \ldots, s_{\ell}$, from the conditional joint distribution $P\left(s_{1}, s_{2}, \ldots, s_{\ell} \mid y\right)$. From our assumption of 286 numerous, independent epistatic terms for each locus, this joint distribution is defined entirely in 287 terms of the means and covariances of the $\ell+1$ variables $y, s_{1}, s_{2}, \ldots, s_{\ell}$. Recall that the fitness 288 effect of a mutation at locus $i$ is $s_{i}=y_{i}-y=-2 \xi_{i}$ as defined in Eq. (10). As shown previously, 289 we have $\overline{s_{i} y}=-2 v_{i}$. We also have $\overline{s_{i} s_{j}}=4 e_{i j}$ with $e_{i i}=v_{i}$. The means of all the variables are 290 zero. Based on this covariance structure, we can compute $P\left(s_{1}, s_{2}, \ldots, s_{\ell} \mid y\right)$ using the properties 291 of the conditional distribution of a multivariate normal distribution. It is straightforward to show 292 that conditional on the fitness $y$, the conditional means are given by $\operatorname{Mean}_{y}\left(s_{i}\right)=-2 v_{i} y$ and the 293 conditional covariances are $\operatorname{Cov}_{y}\left(s_{i}, s_{j}\right)=4\left(e_{i j}-v_{i} v_{j}\right)$. This relation makes clear that in general 294 the DFE from a sample $s_{1}, s_{2}, \ldots, s_{\ell} \sim P\left(s_{1}, s_{2}, \ldots, s_{\ell} \mid y\right)$ depends on the epistatic interactions 295 between all pairs of loci via $e_{i j}$. Note that $\operatorname{Var}_{y}\left(s_{i}\right)=4 v_{i}\left(1-v_{i}\right)$, which leads to Eq. (16) for the 296 marginal distribution $P\left(s_{i} \mid y\right)$ of a particular locus.

The DFE simplifies considerably if we make certain additional assumptions on the nature of 298 epistatic interactions. In particular, the conditional correlation between fitness effects is

$$
\operatorname{Corr}_{y}\left(s_{i}, s_{j}\right)=\frac{e_{i j}-v_{i} v_{j}}{\sqrt{v_{i} v_{j}\left(1-v_{i}\right)\left(1-v_{j}\right)}} .
$$

299 If we assume the typical variance fraction, $\bar{v}$ is small (i.e., $\bar{v} \ll 1$ ) and also that $e_{i j}$ is $O\left(\bar{v}^{2}\right)$, then 
300 correlations are $O(\bar{v})$ and thus negligible. Then, in a particular sample $s_{1}, s_{2}, \ldots, s_{\ell}$, we can think 301 of each $s_{i}$ as being drawn independently with mean $-2 v_{i} y$ and variance $4 v_{i}\left(1-v_{i}\right)$. If $\ell$ is large, 302 then this leads to a DFE

$$
\rho(s \mid y)=\int_{0}^{1} d v(2 \sqrt{v(1-v)})^{-1} P(v) \varphi\left(\frac{s+2 v y}{2 \sqrt{v(1-v)}}\right),
$$

303 where $P(v)$ is the distribution of variance fractions (DVF) across the loci and $\varphi$ is the standard 304 normal pdf. Surprisingly, this relation becomes exact for the CN model, where we have shown 305 that $e_{i j}=v_{i} v_{j}$ (Eq. (50) ) and therefore the correlations between $s_{i}$ 's vanish. In this case, the ${ }_{306} \mathrm{DFE}$ is thus determined entirely by the DVF, but we will show later that it has certain universal 307 properties independent of even the DVF. Note that we derive the DFE starting from rather 308 general assumptions on the organization of the genotype-phenotype map, in contrast to past 309 models which assume the DFE as a starting point.

310 Above, we have measured the DFE for the subset of genotypes that have fitness $y$ without 311 regard to their evolutionary history. Over the course of adaptation, mutations are fixed and 312 certain fitness changes are observed. We would then like to measure the DFE for those genotypes 313 that have undergone a particular adaptive trajectory. As we show below, the DFE again simplifies 314 considerably if certain relations hold.

\section{B. History-dependence}

316 Using an analysis similar to the one in the previous section, we quantify history-dependence 317 by calculating the correlations between the new fitness and the adaptive history conditional on 318 the genotypes that have undergone a specific sequence of events in the past.

To be precise, suppose an initial clonal population of fitness $y_{0}$ gains $k$ successive mutations and the corresponding sequence of fitnesses is $y_{1}, y_{2}, \ldots, y_{k}$. We would like to quantify how the fitness of a new mutation at locus $k+1, y_{k+1}$, depends on past fitnesses and the idiosyncratic epistatic interactions between the previous $k$ mutations and the new mutation. The correlation between any two fitnesses $y_{i}$ and $y_{j}(i<j)$ is given by $1-2 v_{i+1: j}$, where $v_{i+1: j}$ is the variance fraction of the loci $i+1, i+2, \ldots, j$ (the subscript notation will be used throughout). In general, $v_{i+1: j}$ accounts for the epistatic interactions of all orders between these $j-i$ loci and is expressible in terms of the coefficients of our original complex trait model in Eq. (3). One can then write 
the covariance matrix between $y_{0}, y_{1}, \ldots, y_{k+1}$. In block form, this is

$$
\Sigma=\left(\begin{array}{cc}
\Sigma_{0: k, 0: k} & \Sigma_{0: k, k+1} \\
\Sigma_{k+1,0: k} & \Sigma_{k+1, k+1}
\end{array}\right)
$$

319 The mean of $y_{k+1}$ conditional on $y_{0}, y_{1}, \ldots, y_{k}$ is a linear weighted sum of the past fitnesses:

$$
\overline{y_{k+1}}=\Sigma_{k+1,0: k} \Sigma_{0: k, 0: k}^{-1} \boldsymbol{y}
$$

320 where $\boldsymbol{y}$ is a vector with elements $y_{0}, y_{1}, \ldots, y_{k}$. In other words, $y_{k+1}$ can be written as

$$
y_{k+1}=\sum_{i=0}^{k} w_{k+1, i} y_{i}+\epsilon,
$$

where $\epsilon$ is a mean-zero stochastic term that depends on the genotype of the initial population and whose variance can be calculated from $\Sigma$. To gain intuition, it is useful to explicitly calculate the case of $k=1$. In this case, the covariance matrix is

$$
\Sigma=\left(\begin{array}{ccc}
1 & 1-2 v_{1} & 1-2 v_{12} \\
1-2 v_{1} & 1 & 1-2 v_{2} \\
1-2 v_{12} & 1-2 v_{2} & 1
\end{array}\right) .
$$

We have

$$
\begin{aligned}
\Sigma_{2,01} \Sigma_{01,01}^{-1} & =\frac{1}{4 v_{1}\left(1-v_{1}\right)}\left(1-2 v_{12} 1-2 v_{1}\right)\left(\begin{array}{cc}
1 & 2 v_{1}-1 \\
2 v_{1}-1 & 1
\end{array}\right) \\
& =\frac{1}{4 v_{1}\left(1-v_{1}\right)}\left(1-2 v_{12}-\left(1-2 v_{1}\right)\left(1-2 v_{2}\right)\left(1-2 v_{2}\right)-\left(1-2 v_{1}\right)\left(1-2 v_{12}\right)\right) .
\end{aligned}
$$

321 We therefore have

$$
\overline{y_{2}}=\frac{v_{1}-v_{2}+v_{12}-2 v_{1} v_{12}}{2 v_{1}\left(1-v_{1}\right)} y_{1}+\frac{v_{1}+v_{2}-2 v_{1} v_{2}-v_{12}}{2 v_{1}\left(1-v_{1}\right)} y_{0}
$$

322 The dependence on the past has complex dependencies on epistasis between loci 1 and 2 even in 323 this highly simplified case. To identify what contributes to history dependence beyond just the 324 most recent fitness, we re-write Eq. (71) as

$$
\overline{y_{2}}=\left(1-2 v_{2}\right) y_{1}+\frac{\left(1-2 v_{1}\right)\left(v_{12}-v_{1}-v_{2}+2 v_{1} v_{2}\right)}{2 v_{1}\left(1-v_{1}\right)} y_{1}+\frac{v_{1}+v_{2}-2 v_{1} v_{2}-v_{12}}{2 v_{1}\left(1-v_{1}\right)} y_{0} \text {. }
$$


325 From here, we observe that the dependence on $v_{1}$ and $y_{0}$ vanishes precisely when $v_{12}=v_{1}+v_{2}-$ $3262 v_{1} v_{2}$. This is not true for all landscapes. However, as noted previously, this is in fact true for 327 the $\mathrm{CN}$ model (Eq. (50)) when $\bar{\mu} \ell \gg 1$. In this case, we get the simple relation

$$
y_{2}=\left(1-2 v_{2}\right) y_{1}+2 \sqrt{v_{2}\left(1-v_{2}\right)} \eta,
$$

328 where the pre-factor in the second term comes from normalization and $\eta$ is a Gaussian random 329 variable.

330 From Eq. (37), we have the general relation $v_{12}=v_{1}+v_{2}-2 e_{12}$, where $e_{12}$ is the epistatic 331 variance fraction between loci 1 and 2. We can then re-write Eq. (72) as

$$
\overline{y_{2}} \approx\left(1-2 v_{2}\right) y_{1}+\frac{v_{1} v_{2}-e_{12}}{v_{1}} s_{1}
$$

332 for $v_{1} \ll 1$ and $s_{1}=y_{1}-y_{0}$. An intuitive interpretation of this result is presented in the main 333 text.

\section{Sufficient condition for memoryless fitness gains}

The $k=1$ case suggests that the relation for memoryless fitness gains $\left(e_{12}=v_{1} v_{2}\right)$ could in 336 fact be true for all $k$ under the $\mathrm{CN}$ model, which indeed turns out to be the case, as we show 337 below. Motivated by the $k=1$ case, we would like to have $w_{k+1, k}=1-2 v_{k+1}$ in Eq. (67) and 338 the rest of the weights equal to zero. If this is the case,

$$
y_{k+1}=\left(1-2 v_{k+1}\right) y_{k}+\epsilon
$$

339 Multiplying both sides by $y_{j}$ and computing the correlations, we get the condition $1-2 v_{j+1: k+1}=$ $340\left(1-2 v_{j+1: k}\right)\left(1-2 v_{k+1}\right)$ for all $j$. Therefore, a sufficient condition for memoryless fitness gain is

$$
v_{j+1: k+1}=v_{k+1}+v_{j+1: k}-2 v_{k+1} v_{j+1: k}
$$

for all $k$ and for all $j<k$. We will now show that this is true for the CN model for $j=0$, i.e., $v_{1: k+1}=v_{k+1}+v_{1: k}-2 v_{k+1} v_{1: k}$; the rest trivially follows. Let us first analyze what terms contribute towards $V_{1: k}$ (dividing by $V$ gives $v_{1: k}$ ). When loci 1 through $k$ are mutated, their effect is to flip the signs of their coefficients in Eq. (3). The ones which have changed sign contribute to the de-correlation between the fitnesses before and after the set of mutations, but the epistatic 
terms which have an even number of flips do not change and therefore their contribution has to be subtracted. $V_{1: k}$ therefore is the sum of squares of all the coefficients in Eq. (3) whose loci have flipped an odd number of times. To keep track of indices, suppose $i_{1}, i_{2}, \ldots$ are used to denote the indices of the $k$ loci (which take values from 1 to $k$ ) and $j_{1}, j_{2}, \ldots$ for the rest. Then,

$$
\begin{aligned}
V_{1: k} & =\sum_{i_{1}=1}^{k} F_{i_{1}}^{2}+\sum_{i_{1}>i_{2}>i_{3}}^{k} F_{i_{1} i_{2} i_{3}}^{2}+\ldots, \text { where } \\
F_{i_{1}}^{2} & =f_{i_{1}}^{2}+\sum_{j_{1} \neq 1: k} f_{i_{1} j_{1}}^{2}+\sum_{j_{1}>j_{2} \neq 1: k} f_{i_{1} j_{1} j_{2}}^{2}+\ldots, \\
F_{i_{1} i_{2} i_{3}}^{2} & =f_{i_{1} i_{2} i_{3}}^{2}+\sum_{j_{1} \neq 1: k} f_{i_{1} i_{2} i_{3} j_{1}}^{2}+\sum_{j_{1}>j_{2} \neq 1: k} f_{i_{1} i_{2} i_{3} j_{1} j_{2}}^{2}+\ldots,
\end{aligned}
$$

341 and so on. Now, when the $k+1$ th locus is also flipped, to compute $V_{1: k+1}$, we can add up the 342 two variances $V_{1: k}$ and $V_{k+1}$ except for the cross terms which have an even number of sign flips 343 and which include both the $k+1$ th locus and the other $k$ loci. These have to be subtracted twice 344 because they appear both in $V_{1: k}$ and $V_{k+1}$. These terms are

$$
I_{1: k, k+1}=\sum_{i_{1}=1}^{k} F_{i_{1} k+1}^{2}+\sum_{i_{1}>i_{2}>i_{3}}^{k} F_{i_{1} i_{2} i_{3} k+1}^{2}+\ldots
$$

345 where the $F$ s are defined in a similar fashion as in Eq. (77) except the sums over $j$ s run from ${ }_{346} k+2$ to $\ell$ instead of $k+1$ to $\ell$. We get the general relation

$$
V_{1: k+1}=V_{k+1}+V_{1: k}-2 I_{1: k, k+1} \text {. }
$$

For the CN model specifically, we have $\left\langle f_{i_{1} i_{2} \ldots i_{k}}^{2}\right\rangle=\prod_{j=1}^{k} \mu_{i_{j}}$. This implies

$$
\begin{aligned}
\left\langle I_{1: k, k+1}\right\rangle & =\sum_{i_{1}=1}^{k}\left\langle F_{i_{1} k+1}^{2}\right\rangle+\sum_{i_{1}>i_{2}>i_{3}}^{k}\left\langle F_{i_{1} i_{2} i_{3} k+1}^{2}\right\rangle+\ldots, \\
& =\mu_{k+1} \prod_{j=k+2}^{\ell}\left(1+\mu_{j}\right)\left(\sum_{i_{1}=1}^{k} \mu_{i_{1}}+\sum_{i_{1}>i_{2}>i_{3}}^{k} \mu_{i_{1}} \mu_{i_{2}} \mu_{i_{3}}+\ldots\right) \\
& =\mu_{k+1}\left(\prod_{j=k+2}^{\ell}\left(1+\mu_{j}\right)\right)\left(\prod_{i=1}^{k}\left(1+\mu_{i}\right)-1\right)
\end{aligned}
$$

Performing a similar calculation for $\left\langle V_{1: k}\right\rangle$, we find

$$
\begin{aligned}
\left\langle V_{1: k}\right\rangle & =\prod_{j=k+1}^{\ell}\left(1+\mu_{j}\right)\left(\prod_{i=1}^{k}\left(1+\mu_{i}\right)-1\right), \\
& =\left(1+\mu_{k+1}\right)\left(\prod_{j=k+2}^{\ell}\left(1+\mu_{j}\right)\right)\left(\prod_{i=1}^{k}\left(1+\mu_{i}\right)-1\right)
\end{aligned}
$$


347 which gives

$$
\left\langle I_{1: k, k+1}\right\rangle=v_{k+1}\left\langle V_{1: k}\right\rangle .
$$

348 for $\mu \ell \gg 1$ since $v_{k+1}=\mu_{k+1} /\left(1+\mu_{k+1}\right)$. Dividing Eq. (79) by $V$ throughout concludes the 349 derivation.

\section{Adaptedness}

Under the conditions of memoryless fitness gains from the previous section, we can write

$$
\sigma=-2 v z+2 \sqrt{v(1-v)} \nu
$$

352 where variables have been re-scaled as $z=V^{-1 / 2}(y-\bar{y}), \sigma=V^{-1 / 2} s, \nu=V^{-1 / 2} \eta$. This equation 353 suggests various forms for the DFE, $\rho(\sigma \mid z)$, depending on the DVF, $P(v)$.

$354 \quad z$ is an intuitive measure of "adaptedness": (1) When $z$ is negative, the organism is in the 355 unlikely situation of being "negatively-adapted" to the environment. Beneficial mutations are 356 much more likely than deleterious mutations and adaptation is dominated by loci that have a 357 large $v$. (2) When $|z| \ll 1$, the organism is "neutrally-adapted". The number of beneficial and 358 deleterious mutations are balanced. (3) When $z \gg 1$, the organism is "well-adapted", where the 359 DFE is strongly skewed towards deleterious mutations.

We will analyze adaptation in the neutrally-adapted and the well-adapted regimes. When the 361 organism is negatively-adapted, Eq. (83) predicts that a few substitutions are sufficient to quickly 362 reach the neutrally-adapted state. In addition, we assume the variance fraction of each locus is 363 small, i.e., $v \ll 1$ but the number of loci $\ell$ is large enough so that overall epistasis $\approx \bar{v} \ell \gg 1(\bar{v}$ is 364 the mean variance fraction). We can then ignore the $1-v$ factor and re-write Eq. (83) as

$$
\sigma=-2 v z+2 \sqrt{v} \nu
$$

365 The intuition behind the analytical results is discussed in the main text. We present the formal 366 calculations here.

\section{E. Analytical results for an exponential DVF}

We begin by calculating the DFE, $\rho(\sigma \mid z)$, for the specific case when the DVF is an exponential,

$$
P(v)=\bar{v}^{-1} e^{-v / \bar{v}}
$$


369 where $\bar{v} \ll 1$ is the mean variance fraction across loci. The true DVF likely has a large fraction 370 of loci that have no effect; accounting for these loci simply scales the mutation rate and will be 371 ignored in this analysis. The exponential DVF leads to analytical predictions for the shape of the 372 average fitness trajectories under certain simplifying assumptions about the underlying selective 373 forces.

374 From Eq. (84), we have

$$
\rho(\sigma \mid z)=\iint d v d \nu P(v) P(\nu) \delta(\sigma+2 v z-2 \sqrt{v} \nu)
$$

375 Here $\nu$ is a standard normal random variable, which we integrate out, giving

$$
\rho(\sigma \mid z)=\int_{0}^{1} d v(2 \sqrt{v})^{-1} P(v) \varphi\left(\frac{\sigma+2 v z}{2 \sqrt{v}}\right)
$$

376 where $\varphi$ is the normal pdf. For $P(v)$ given in Eq. (85), this integral can be calculated exactly:

$$
\rho(\sigma \mid z)=\frac{\bar{v}^{-1}}{2 \sqrt{2 \bar{v}^{-1}+z^{2}}} e^{-\sigma z / 2-|\sigma| \sqrt{2 \bar{v}^{-1}+z^{2}} / 2} .
$$

377 The resulting DFE is a double exponential with scale $\sim\left(z / 2 \pm \sqrt{2 \bar{v}^{-1}+z^{2}} / 2\right)^{-1}$. For $|z| \ll 1$ small, 378 the DFE is symmetric around the origin, as expected, with a scale determined by the DVF. As $379 z$ increases, the DFE skews towards deleterious effects. The typical magnitudes of beneficial and 380 deleterious effects are not independent from the overall ratio of beneficial to deleterious mutations. 381 The well-adapted regime is reached when $z^{2}$ is comparable to $\bar{v}^{-1}$. To clearly delineate the two 382 regimes, it is useful to define new variables $x=z \sqrt{\bar{v} / 2}$ and $\lambda=\sigma \sqrt{2 / \bar{v}}$. In the new variables, 383 the DFE is

$$
\rho(\lambda \mid x)=\frac{1}{4 \sqrt{1+x^{2}}} e^{-\frac{\lambda x+|\lambda| \sqrt{1+x^{2}}}{2}} .
$$

The neutrally-adapted and well-adapted regimes then correspond to $x \lesssim 1$ and $x \gtrsim 1$ respectively. The mean rate of adaptation in units of generations on the $x$ scale is $\langle d x\rangle=\langle d z\rangle \sqrt{\bar{v} / 2}=$ $\langle\sigma\rangle \sqrt{\bar{v} / 2}=\langle\lambda\rangle \bar{v} / 2$, where the expectation is taken over fixation probabilities and the DFE. We assume strong-selection-weak-mutation $(\mathrm{SSWM})$ so that $p_{\text {fix }}(\sigma) \sim 2 \sigma$ or $p_{\text {fix }}(\lambda) \sim 2 \lambda \sqrt{\bar{v} / 2}$ for positive $\lambda$ and 0 otherwise. We find

$$
\begin{aligned}
\langle d x\rangle & =N U \bar{v} / 2 \int_{-\infty}^{\infty} d \lambda p_{\text {fix }}(\lambda) \rho(\lambda \mid x) \lambda \\
& =N U \bar{v} \sqrt{\bar{v} / 2} \int_{0}^{\infty} d \lambda \lambda^{2} \rho(\lambda \mid x),
\end{aligned}
$$


384 where $N$ is population size and $U$ is the effective mutation rate for loci with non-zero effect. 385 Integrating over $\lambda$, we get

$$
\langle d x\rangle=\frac{N U(2 \bar{v})^{3 / 2}}{\sqrt{1+x^{2}}\left(x+\sqrt{1+x^{2}}\right)^{3}} .
$$

Integrating over $d x$ starting from an initial $x_{0}$, we obtain an approximation to the mean fitness trajectory

$$
\begin{aligned}
& N U(2 \bar{v})^{3 / 2} n_{\text {gen }}=\int_{x_{0}}^{x} d x \sqrt{1+x^{2}}\left(x+\sqrt{1+x^{2}}\right)^{3}=T(x)-T\left(x_{0}\right), \\
& \text { where } T(x)=\frac{4 x^{5}}{5}+\frac{5 x^{3}}{3}+\frac{\sqrt{x^{2}+1}}{15}\left(12 x^{4}+19 x^{2}+7\right)+x .
\end{aligned}
$$

386 Note that this is only an approximation for small $x$ since the typical fixed beneficial effect is 387 a discrete jump. We show the result from Eq. (93) in Figure 11a, where the dependencies on 388 the equilibrium fitness $\bar{y}$ and genetic variance $V$ are highlighted. There are two independent 389 parameters that determine the scale $(\sqrt{\bar{v} / 2 V})$ and location $(\bar{y})$ of the fitness, and one parameter $390\left(N U n_{\text {gen }}\right)$ that determines the time scale. From Eq. (93), the $x \sim t$ and $x \sim t^{1 / 5}$ scalings in the 391 neutrally-adapted $(x \ll 1)$ and well-adapted regime $(x \gg 1)$ respectively are apparent.

The number of substitutions, $n_{s}$, as a function of $x$ can also be calculated under the SSWM assumption. We get

$$
\begin{aligned}
& \bar{v} n_{s}=N(x)-N\left(x_{0}\right), \\
& \text { where } \quad N(x)=x\left(x+\sqrt{x^{2}+1}\right) / 4+\sinh ^{-1}(x) / 4,
\end{aligned}
$$

392 which can be mapped onto time using Eq. (93). The scalings are $n_{s} \sim x \sim t$ in the neutrally393 adapted regime and $n_{s} \sim x^{2} \sim t^{2 / 5}$ in the well-adapted regime.

\section{F. Asymptotics and general scaling results}

We now derive the asymptotic properties of the DFE in the well-adapted regime $\left(\bar{v}^{1 / 2} z \gg 1\right)$. Writing out the Gaussian pdf in Eq. (64), we have

$$
\begin{aligned}
\rho(\sigma \mid z) & =\int_{0}^{1} d v(2 \sqrt{2 \pi v})^{-1} P(v) e^{-\frac{(\sigma+2 v z)^{2}}{8 v}} \\
& =e^{-\sigma z / 2} \int_{0}^{1} d v(2 \sqrt{2 \pi v})^{-1} P(v) e^{-\frac{\sigma^{2}}{8 v}-\frac{v z^{2}}{2}} \\
& =e^{-\sigma z / 2} \int_{0}^{1} d u(2 \sqrt{2 \pi u y})^{-1} P(u / z) e^{-\frac{z}{2}\left(\frac{\sigma^{2}}{4 u}+u\right)},
\end{aligned}
$$


395 where the change of variables $v=u / z$ is used. When $z \gg 1$, Laplace's method can be used. The 396 exponent is minimized when $u=|\sigma| / 2$, which gives

$$
\rho(\sigma \mid z) \approx(2 z)^{-1} P(|\sigma| / 2 z) e^{-\frac{z}{2}(\sigma+|\sigma|)}
$$

${ }_{397}$ The contribution towards a fitness effect $\sigma$ at large $z$ comes largely from loci with variance fraction з98 $v \approx|\sigma| / 2 z$. The exponential form of the beneficial DFE is determined entirely by the Gaussian 399 tails of the genotype-dependent term. The argument can be easily generalized to non-Gaussian 400 tail probabilities using a similar calculation. Eq. (100) implies that the ratio of the probabilities 401 of beneficial and deleterious mutations is independent of the DVF as long as it has sufficient mass 402 at $v=|\sigma| / 2 z$ :

$$
\frac{\rho(\sigma \mid z)}{\rho(-\sigma \mid z)}=e^{-\sigma z}
$$

${ }_{403}$ Such a relationship has been hypothesized previously based on simulations of an additive finite${ }_{404}$ sites model and the form of $p_{\text {fix }}$ close to the high-interference limit that could result in a fitness 405 plateau [6]. Using our theory, we have shown that this result is indeed true independent of 406 the DVF and under our core hypotheses of normality and memoryless fitness gains. If $p_{\text {fix }} \sim$ $407 e^{\sqrt{V} T_{c} \sigma}$ in the high-interference limit, where $T_{c}$ is the coalescent time, fitness should plateau when ${ }_{408} p_{\text {fix }}(\sigma) \rho(\sigma \mid z)=p_{\text {fix }}(-\sigma) \rho(-\sigma \mid z)$, which is at

$$
z_{\text {plateau }}=2 \sqrt{V} T_{c}
$$

409 The $\sqrt{V}$ appears to account for the re-scaling $\sigma=s / \sqrt{V}$.

410 We get Eq. (100) only if $P(v)$ has probability mass at $v=|\sigma| / 2 z$. This is not the case in 411 the exponentially-correlated fitness landscape model with homogeneous loci, i.e., if for instance ${ }_{412} P(v)=\delta(v-\bar{v})$, we instead get from Eq. (64)

$$
\rho(\sigma \mid z)=\varphi\left(\frac{\sigma+2 \bar{v} z}{2 \sqrt{\bar{v}}}\right) .
$$

${ }_{413}$ The DFE in this case is therefore a Gaussian with mean shifting towards the deleterious side. The ${ }_{414}$ ratio of beneficial to deleterious mutations goes rapidly to zero as $\simeq \varphi(\sqrt{\bar{v}} z) / \sqrt{\bar{v}} z$ and adaptation 415 sharply plateaus beyond $z=\bar{v}^{-1 / 2}$.

From Eq. (100) various scaling results can be derived that apply independent of $P(v)$ and the Gaussian assumption on $\nu$. We retain the exponential form for convenience. The rate of beneficial 
mutations is

$$
\begin{aligned}
U_{b} & =U \int_{0}^{\infty} d \sigma(2 z)^{-1} P(|\sigma| / 2 z) e^{-\sigma z} \\
& =\frac{U}{2 z^{2}} \int_{0}^{\infty} d w P\left(w / 2 z^{2}\right) e^{-w} .
\end{aligned}
$$

${ }_{416}$ The integral has an effective upper cutoff at $w \sim O(1)$ and can be approximated as $U_{b} \approx$ ${ }_{417} U P(0) / 2 z^{2} \sim z^{-2}$ under certain assumptions for $P(v)$ for small $v$. The pre-factor $P(0)$ suggests ${ }_{418}$ that the number of beneficial mutations depends on the number of small-effect loci. While strong ${ }_{419}$ epistatic loci drive adaptation in the neutrally-adapted regime, adaptation in the well-adapted 420 regime is instead driven by weakly epistatic loci.

${ }_{421}$ From Eq. 100 , the typical size of a beneficial mutation is $\sigma \sim z^{-1}$. Under SSWM, $p_{\text {fix }} \sim \sigma \sim$ ${ }_{422} z^{-1}$. As argued previously, since $U_{b} \sim z^{-2}$, we get $d z / d t \sim z^{-4}$ and therefore we obtain the two 423 scaling relations

$$
z \sim t^{1 / 5}, n_{s} \sim t^{2 / 5}
$$

424 which apply independently of the form of the DVF in the well-adapted regime.

425 [1] M. S. Johnson, A. Martsul, S. Kryazhimskiy, and M. M. Desai, Science 366, 490 (2019).

426 [2] S. Hwang, B. Schmiegelt, L. Ferretti, and J. Krug, Journal of Statistical Physics 172, 226 (2018).

427 [3] P. F. Stadler and R. Happel, Journal of Mathematical Biology 38, 435 (1999).

428 [4] J. Neidhart, I. G. Szendro, and J. Krug, Journal of theoretical biology 332, 218 (2013).

429 [5] A. Agarwala and D. S. Fisher, Theoretical population biology 130, 13 (2019).

430 [6] D. P. Rice, B. H. Good, and M. M. Desai, Genetics 200, 321 (2015). 

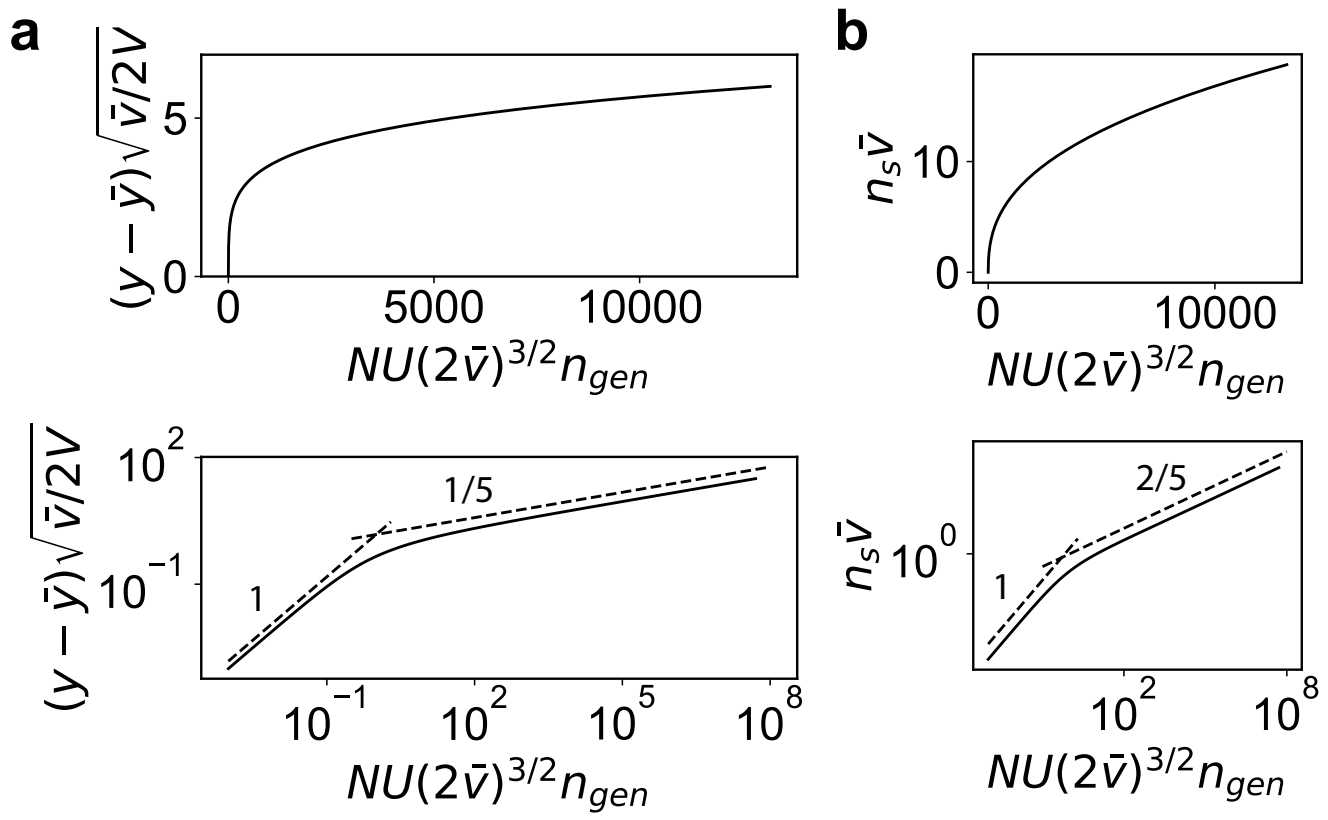

Figure 1. The mean fitness trajectory and the mean number of substitutions predicted by the model in the strong-selection-weak-mutation regime. (a) The scaled fitness vs scaled time. Shown below is the fitness in log-scale to highlight the different scalings in the neutrally-adapted and well-adapted regimes. The slopes of the dashed lines are shown. (b) The scaled number of substitutions vs scaled time as in panel (a). 